\title{
Dimensional crossover in quantum critical metallic magnets
}

\author{
Markus Garst, ${ }^{1}$ Lars Fritz, ${ }^{2}$ Achim Rosch, ${ }^{1}$ and Matthias Vojta ${ }^{1}$ \\ ${ }^{1}$ Institut für Theoretische Physik, Universität zu Köln, Zülpicher Str. 77, 50937 Köln, Germany \\ ${ }^{2}$ Department of Physics, Harvard University, Cambridge MA 02138, USA
}

(Dated: October 30, 2018)

\begin{abstract}
Nearly magnetic metals often have layered lattice structures, consisting of coupled planes. In such a situation, physical properties will display, upon decreasing temperature or energy, a dimensional crossover from two-dimensional (2d) to three-dimensional (3d) behavior, which is particularly interesting near quantum criticality. Here we study this crossover in thermodynamics using a suitably generalized Landau-Ginzburg-Wilson approach to the critical behavior, combined with renormalization group techniques. We focus on two experimentally relevant cases: the crossover from a $2 \mathrm{~d}$ to a $3 \mathrm{~d}$ antiferromagnet, and the crossover from a $2 \mathrm{~d}$ ferromagnet to a $3 \mathrm{~d}$ antiferromagnet. As naive scaling does not apply at and above the upper critical dimension, two crossover scales arise which can be associated with separate dimensional crossovers of classical and quantum fluctuations, respectively. In particular, we find an intermediate regime with novel power laws where the quantum fluctuations still have a $2 \mathrm{~d}$ and the classical fluctuations already have a $3 \mathrm{~d}$ character. For the ferromagnet-to-antiferromagnet crossover, the mismatch of the dynamical exponents between the $2 \mathrm{~d}$ and $3 \mathrm{~d}$ regimes leads to an even richer crossover structure, with an interesting $2 \mathrm{~d}$ non-critical regime sandwiched between two critical regimes. For all cases, we find that thermal expansion and compressibility are particularly sensitive probes of the dimensional crossover. Finally, we relate our results to experiments on the quantum critical heavy-fermion metals $\mathrm{CeCu}_{6-x} \mathrm{Au}_{x}, \mathrm{YbRh}_{2} \mathrm{Si}_{2}$, and $\mathrm{CeCoIn}_{5}$.
\end{abstract}

PACS numbers: 71.27.+a,71.10.Hf

\section{INTRODUCTION}

Quantum phase transitions (QPT) in metals are a fascinating field of today's condensed matter research. $\frac{1}{2}$ Heavy-fermion materials play a prominent role: Frequently, one observes non-Fermi liquid behavior which is thought to be associated with an antiferromagnetic (AFM) instability of the itinerant electrons. The critical spin fluctuations near the phase transition lead to unconventional power laws in transport and thermodynamic quantities at low temperatures. On the theory side, a Landau-Ginzburg-Wilson (LGW) description of the critical magnetic degrees of freedom, developed by

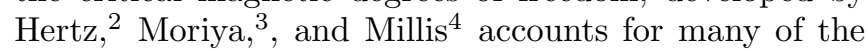
experimental signatures of magnetic criticality.

However, some heavy-fermion compounds do not easily fit in the LGW picture in $d=3$ spatial dimensions. For $\mathrm{CeCu}_{6-x} \mathrm{Au}_{x}$, it was realized ${ }^{5}$ that most thermodynamic signatures of the QPT at $x=0.1$ are consistent with the assumption of the underlying AFM spin fluctuations to be $2 \mathrm{~d}$. It came as a surprise that $2 \mathrm{~d}$ spin fluctuations should prevail in an intrinsically $3 \mathrm{~d}$ alloy, but the $2 \mathrm{~d}$ character was subsequently confirmed in neutronscattering experiments ${ }^{6}$ Importantly, the AFM order observed in $\mathrm{CeCu}_{6-x} \mathrm{Au}_{x}$ below the Néel temperature is fully 3d. On general grounds, one thus expects a dimensional crossover within the paramagnetic phase from $2 \mathrm{~d}$ magnetic fluctuations at elevated temperatures to $3 \mathrm{~d}$ fluctuations at lowest temperatures or in the immediate vicinity of the phase transition. Experimentally, the dimensional crossover in $\mathrm{CeCu}_{6-x} \mathrm{Au}_{x}$ has proven to be elusive so far.
A related heavy-fermion metal, not easily fitting the LGW theory framework, is $\mathrm{YbRh}_{2} \mathrm{Si}_{2}{ }^{\underline{7}}$ It shows a phase transition to an ordered phase at $70 \mathrm{mK}$, which is believed to be AFM, however, a confirmation by neutron scattering is not available to date. An additional aspect is that $\mathrm{YbRh}_{2} \mathrm{Si}_{2}$ seems to be almost ferromagnetic $(\mathrm{FM}) \stackrel{8}{\frac{8}{9}}$ and we will return to this issue later in this paper. The unusual properties of both $\mathrm{CeCu}_{6-x} \mathrm{Au}_{x}$ and $\mathrm{YbRh}_{2} \mathrm{Si}_{2}$ have prompted speculations on the inapplicability of the LGW theory, which describes a magnetic instability of well-defined quasiparticles: Instead, it was proposed that the Kondo effect, being responsible for the formation of the heavy quasiparticles, breaks down at the quantum critical point (QCP) $\stackrel{9,10}{=}$ Different scenarios and theoretical descriptions of this Kondo breakdown have been put forward $\stackrel{9,10,11}{=}$ The scenario of so-called "local quantum criticality" $\underline{\underline{9}}$ uses an extension of dynamical mean-field theory to map the Kondo-lattice problem to a self-consistent impurity model where the Kondo effect may be suppressed by critical bulk spin fluctuations. This particular scenario for a Kondo-breakdown QCP requires the spin fluctuations to be $2 \mathrm{~d}$; for $3 \mathrm{~d}$ spin fluctuations this model predicts a conventional magnetic QCP of LGW type. As the critical spin fluctuations of the material can again be expected to become $3 \mathrm{~d}$ at low energies, the local quantum criticality should be restricted to elevated temperatures or energies above the dimensional crossover.

Other examples of layered metals with magnetic QCP are the heavy fermions $\mathrm{CeMIn}_{5}(\mathrm{M}=\mathrm{Co}, \mathrm{Rh}, \mathrm{Ir})$, the hightemperature superconducting cuprates and iron pnictides, and the metamagnetic ruthenate $\mathrm{Sr}_{2} \mathrm{Ru}_{3} \mathrm{O}_{7}$.

The purpose of this paper is to study theoretically the 
dimensional crossover of critical magnetic fluctuations in the framework of the LGW model, with focus on the 2d AFM to 3d AFM and 2d FM to 3d AFM crossovers. Microscopically, we imagine a system of planes of interacting electrons, with tendency toward antiferromagnetic or ferromagnetic in-plane ordering, and a weak antiferromagnetic inter-plane coupling. We will study how the dimensional crossover is reflected in the correlation length, specific heat, thermal expansion, compressibility, and the Grüneisen parameter $\stackrel{12}{=}$ Our primary goal is to identify observables which are suited for an experimental search for a dimensional crossover.

In this paper, we shall refrain from a detailed microscopic modeling of the magnetic inter-plane coupling. The underlying lattice geometry and bandstructure will influence some of the non-universal properties of the dimensional crossover; however, the existence of welldefined $2 \mathrm{~d}$ and $3 \mathrm{~d}$ regimes is independent of those details. We shall ignore complications arising from the possibility of the inter-plane coupling being frustrated:13,14,15 Even for a fully frustrated coupling, a dimensional crossover to $3 \mathrm{~d}$ behavior at low energies will generically occur, albeit with a possibly small crossover scale $\stackrel{14,15}{1}$ (In situations with frustration, the effective $3 \mathrm{~d}$ coupling within the ordered phase may be enhanced due to order-from-disorder mechanisms.)

We shall restrict our analysis to the framework of the LGW theory of itinerant spin fluctuations. It has been discussed that this approach may break down at lowest energies due to the occurrence of singular terms in the LGW expansion (i) for ferromagnets in both $2 \mathrm{~d}$ and $3 \mathrm{~d}, \stackrel{16}{=}$ and (ii) for $2 \mathrm{~d}$ antiferromagnets,$\frac{17}{2}$ These complications will be ignored for simplicity, a justification being that the low-energy behavior of our model is invariably 3d AFM (where the LGW approach is believed to be valid) $\stackrel{18}{=}$ Our theory may also be combined with the ideas of local quantum criticality,$\underline{9}$ this is beyond the scope of this paper. Similarly, an explicit treatment of the magnetically ordered phases shall not be performed here, 19,20

\section{A. Summary of results}

The main results of our analysis are the crossover phase diagrams, Figs. 1 and 2, for the 2d - 3d AFM and the 2d FM - 3d AFM crossover, respectively. The large anisotropy in the spin-fluctuation spectrum defines a small momentum scale $\Lambda_{\eta}$ [see Eq. (6) below] that determines the positions of the dimensional crossovers in the phase diagram, as indicated by the shaded areas. Generally, the behavior changes from $2 \mathrm{~d}$ to $3 \mathrm{~d}$ upon approaching criticality.

However, the fact that the QPT under consideration are at or above their upper critical dimension renders naive scaling invalid; as a result, the quantum critical regime is characterized by two distinct length scales: The physical correlation length $\xi$ and a thermal length given by $T^{-1 / z}$, where $T$ is the temperature and $z$ the dynam-

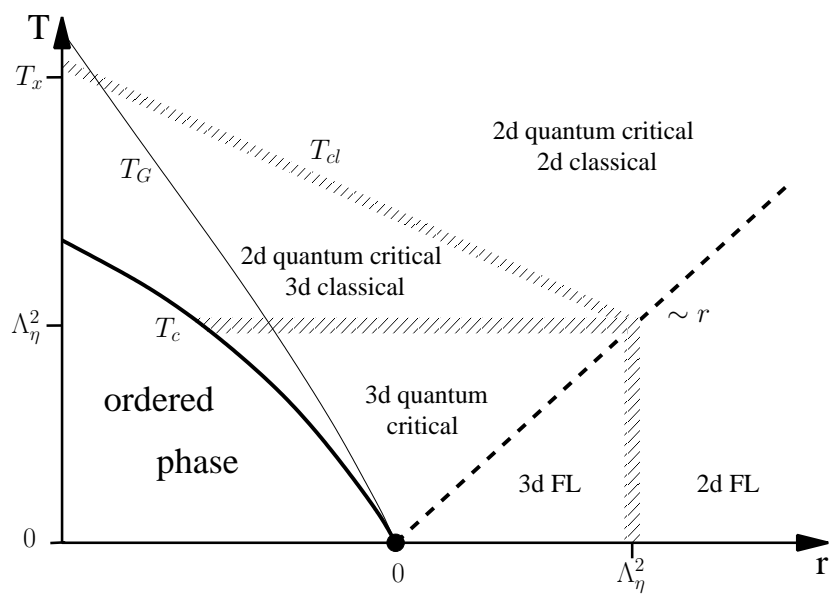

FIG. 1: Phase diagram of the anisotropic LGW model in the temperature-control parameter plane for the $2 \mathrm{~d}$ AFM to $3 \mathrm{~d}$ AFM crossover. The crossover scale is determined by the momentum scale $\Lambda_{\eta}$. The $3 \mathrm{~d}$ spin fluctuations dominate close to the quantum critical point. A dimensional crossover occurs upon increasing the distance to the QCP indicated by the shaded region. The phase boundary $T_{c}(r)$ changes its behavior at this crossover, see Fig. 4 below. There is an additional dimensional crossover at the temperature scale $T_{c l}(r)$ where $\xi \sim 1 / \Lambda_{\eta}$, associated with the classical critical fluctuations. The thin line, $T_{G}(r)$, close to the critical temperature, $T_{c}(r)$, indicates the Ginzburg temperature where the crossover to classical Wilson-Fisher behavior occurs. The two lines $T_{c l}(r)$ and $T_{G}(r)$ cross at a temperature $T_{x}$. The dashed line separates the low-temperature magnetically disordered (Fermiliquid, FL) regime, $T \ll r$, from the quantum critical regime, $T \gg r$. Note that the labels " $2 \mathrm{~d}$ " and " $3 \mathrm{~d}$ " refer to the behavior of the critical or near-critical spin fluctuations, for details see text.

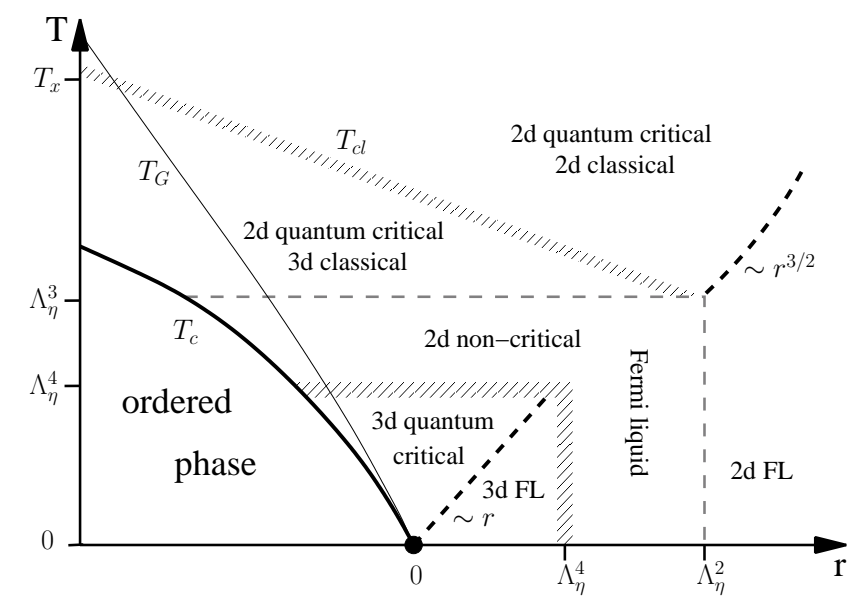

FIG. 2: Phase diagram of the anisotropic LGW model as in Fig. 1 but for the $2 \mathrm{~d}$ FM to $3 \mathrm{~d}$ AFM crossover. Here, the critical 2d regime, $T>\Lambda_{\eta}^{3}$ or $r>\Lambda_{\eta}^{2}$, is separated from the critical 3d regime, $T<\Lambda_{\eta}^{4}$ and $r<\Lambda_{\eta}^{4}$, by a region where $2 \mathrm{~d}$ non-critical Fermi liquid behavior prevails. 
ical exponent. The presence of two length scales results in two types of dimensional crossovers.

\section{Quantum crossover}

A dimensional crossover in the quantum critical fluctuations occurs upon approaching the QCP either by lowering the temperature $T$ or decreasing the tuning parameter $r$; these crossovers are indicated by the horizontal and vertical shaded regions in Figs. 1 and 2

In the case of the $2 \mathrm{~d}$ to $3 \mathrm{~d}$ AF crossover, the positions of these crossover lines are given by $T \sim \Lambda_{\eta}^{z}$ and $r \sim \Lambda_{\eta}^{1 / \nu}$, where $z=2$ and the correlation-length exponent has the mean-field value $\nu=1 / 2$. For example, the specific heat coefficient at criticality, $r=0$, changes its temperature dependence at the crossover temperature, $T \sim \Lambda_{\eta}^{2}$, from $\gamma \sim \log (1 / T)$ to $\gamma \sim$ const $-\sqrt{T}$, as expected for critical thermodynamics of $2 \mathrm{~d}$ and $3 \mathrm{~d}$ AF fluctuations, respectively.

The situation is more complicated for the $2 \mathrm{~d}$ FM to 3d AFM crossover, due to the mismatch of dynamical exponents in the two regimes. The low dimensionality, $d=2$, of the FM spin fluctuations combined with a large dynamical exponent, $z=3$, result in strong thermodynamic signatures of the $2 \mathrm{~d}$ regime, which dominate over the $3 \mathrm{~d}$ AFM fluctuations in an unexpectedly wide regime. For example, at criticality, $r=0$, the $2 \mathrm{~d}$ FM fluctuations yield singular quantum critical thermodynamics down to a temperature scale $T \sim \Lambda_{\eta}^{z}$, where $z=3$. Below this temperature scale, the thermal activation of these $2 \mathrm{~d}$ fluctuations only yields Fermi-liquid behavior, but this is still much stronger than the contribution from the $3 \mathrm{~d}$ part of the spin-fluctuation spectrum. Only at a much lower temperature scale, $T \sim \Lambda_{\eta}^{4}$, the contributions from the $3 \mathrm{~d}$ AFM fluctuations finally take over. Thus, we have here the peculiar situation that a $2 \mathrm{~d}$ non-critical Fermi-liquid regime is sandwiched between the $2 \mathrm{~d}$ and $3 \mathrm{~d}$ quantum critical regions.

\section{Classical crossover}

In addition, there is a dimensional crossover associated with classical criticality. Upon approaching the classical phase transition line, $T_{c}(r)$, the correlation length $\xi$ increases. If $\xi$ reaches $1 / \Lambda_{\eta}$, the classical fluctuations (i.e. those associated with zero Matsubara frequency) effectively change their dimensionality from $2 \mathrm{~d}$ to $3 \mathrm{~d}$. This classical dimensional crossover occurs within the $2 \mathrm{~d}$ quantum critical regime at the shaded line labeled $T_{c l}(r)$ in Figs. 1 and 2, and it also causes thermodynamic signatures. For example, the thermal expansion $\alpha(T)$ has a maximum at $T_{c l}$ for the $2 \mathrm{~d} F M$ to $3 \mathrm{~d}$ AFM crossover. At higher temperature $T_{x}$, the crossover line $T_{c l}$ enters the Ginzburg regime of the classical transition, and the classical dimensional crossover becomes non-perturbative.

\section{Phase boundary and QCP location}

The phase boundary, $T_{c}(r)$, of the classical transition is linear in the distance to the QCP at elevated temperatures (with logarithmic corrections), but curves towards the QCP in the $3 \mathrm{~d}$ regime. As a consequence, an extrapolation of the quasi-linear phase boundary in the $2 \mathrm{~d}$ regime towards zero temperature yields an incorrect position for the QCP, see Fig. 4, and we estimate the corresponding error between the extrapolated and the true position of the QPT.

We note that our treatment of thermodynamics is limited to the non-ordered side of the phase transition and, in particular, breaks down upon entering the Ginzburg regime of the classical critical transition indicated by the thin line $T_{G}(r)$ in Figs. 1 and 2

\section{Observables}

As detailed below, we find that the thermal expansion and the compressibility are well suited to detect a dimensional crossover in the spin-fluctuation spectrum. Both possess pronounced signatures close to the expected crossovers as a function of temperature in the quantum critical regime, either a sharp drop or even a maximum. In contrast to this, the specific heat coefficient only shows a leveling off upon entering the $3 \mathrm{~d}$ regime, which is harder to identify experimentally, see Figs. 5 and 6 below.

\section{B. Outline}

The body of the paper is organized as follows: In Sec. II] we introduce the Landau-Ginzburg-Wilson field theory for magnetism near quantum criticality. We discuss the anisotropic spin susceptibility and the associated crossover in the Landau damping. We introduce our model for the dimensional crossover and give the resulting formulae that determine the correlation length and other thermodynamic properties. Secs. III and IV are devoted to a detailed discussion of the $2 \mathrm{~d}-3 \mathrm{~d}$ AFM and $2 \mathrm{~d}$ FM - 3d AFM crossovers, respectively. We shall derive phase diagrams and full crossover functions for thermodynamic quantities. Finally, in Sec. $\mathrm{V}$ we discuss existing experimental data vis-a-vis our theory results. We focus on the heavy-fermion metals $\mathrm{CeCu}_{6-x} \mathrm{Au}_{x}, \mathrm{YbRh}_{2} \mathrm{Si}_{2}$, and $\mathrm{CeCoIn}_{5}$ which indeed display unconventional quantum criticality that may originate from quasi- $2 \mathrm{~d}$ spin fluctuations. A brief outlook concludes the paper.

\section{ORDER-PARAMETER FIELD THEORY FOR SPATIALLY ANISOTROPIC SPIN FLUCTUATIONS}

In order to analyze the dimensional crossover we will use the standard LGW critical theory of Hertz, Millis 


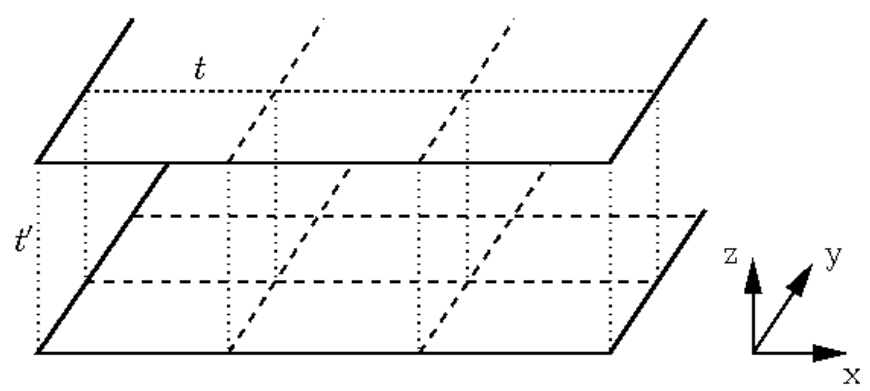

FIG. 3: Schematics of a layered crystal structure with a weak hopping of spin-fluctuations between the planes, $t^{\prime} \ll t$.

and Moriya for a (commensurate) itinerant paramagnet. The action of the Hertz-Millis-Moriya model reads $1,2,4$

$$
\mathcal{S}=\int_{0}^{\beta} d \tau \int d^{d} \mathbf{r}\left[\frac{1}{2} \Phi^{T} \chi_{0}^{-1}\left(-i \nabla, \partial_{\tau}\right) \Phi+\frac{u_{0}}{4 !}\left(\Phi^{T} \Phi\right)^{2}\right] .
$$

where the real bosonic order-parameter field $\Phi$ represents commensurate spin fluctuations with a $3 \mathrm{~d}$ ordering wavevector Q. We will generalize the field $\Phi$ to have $N$ components; the Heisenberg paramagnet corresponds to $N=3$. The dynamics of the fluctuations are encoded in the propagator $\chi_{0}^{-1}$. Its momentum dependence will reflect the spatial anisotropy of the spin-fluctuation spectrum. Its form will be motivated in the following.

\section{A. Bare susceptibility}

Starting from a model of interacting electrons on a 3d anisotropic lattice, a Hubbard-Stratonovich transformation allows to introduce collective-mode variables representing the spin-fluctuations. Their dispersion can be estimated, e.g., by RPA. Consider for simplicity a paramagnon dispersion of tight-binding type. On a $3 \mathrm{~d}$ tetragonal lattice, a generic form is

$$
\omega(\mathbf{k})=t\left(2-\cos k_{x} a-\cos k_{y} a\right)+t^{\prime}\left(1-\cos k_{z} a\right)
$$

where $t$ and $t^{\prime}$ parameterize the hopping of the spin fluctuations within and perpendicular to the $x y$ planes, see Fig. 3. the momentum $\mathbf{k}$ is measured relative to the ordering wavevector $\mathbf{Q}$, and $a$ is a lattice constant. The quasi-2d character of the spin fluctuations is reflected in a small ratio between the hopping amplitudes,

$$
\eta \equiv t^{\prime} / t \ll 1 \text {. }
$$

Near the transition we can restrict ourselves to energies much smaller than the (large) in-plane bandwidth $\propto t$; this is equivalent to a continuum approximation w.r.t. the in-plane lattice coordinates. The result is

$$
\omega(\mathbf{k})=t \xi_{0}^{2}\left(k_{\|}^{2}+\frac{2 \eta}{a^{2}}\left(1-\cos k_{z} a\right)\right)
$$

where $\xi_{0}=a / \sqrt{2}$ and $k_{\|}$is the in-plane momentum. (More generally, $\xi_{0}$ is a microscopic length scale of order $1 / k_{F}$ where $k_{F}$ is the electronic Fermi momentum.)

The character of the spin fluctuations depends on whether their energy is larger or smaller than the vertical bandwidth $t^{\prime}$. In the energy range $t^{\prime} \ll \omega \ll t$, the paramagnon excitation energy is mainly accounted for by the in-plane momentum $k_{\|}$, and in this sense the spin fluctuations are effectively two-dimensional,

$$
\omega(\mathbf{k}) \approx t \xi_{0}^{2} k_{\|}^{2}, \quad \text { for } \quad t^{\prime} \ll \omega \ll t \quad(2 \mathrm{~d}) .
$$

The restriction on the energy $\omega$ implies that the in-plane momenta are confined to the window $\Lambda_{\eta} \ll k_{\|} \ll \Lambda$, where $\Lambda \sim \xi_{0}^{-1}$ is a momentum cutoff, and we defined for later convenience the crossover momentum

$$
\Lambda_{\eta}=\sqrt{\eta} \Lambda .
$$

For such large energies, $\omega \gg t^{\prime}$, the paramagnons are effectively dispersionless in the direction perpendicular to the planes, such that fluctuations with all vertical momenta $k_{z}$ are equivalent. On the other hand, for energies of order $t^{\prime}$ and smaller, the vertical momentum can be resolved, and the spin fluctuations have a $3 \mathrm{~d}$ character. For $\omega \ll t^{\prime}$ a full continuum approximation is justified, i.e.

$$
\omega(\mathbf{k}) \approx t \xi_{0}^{2}\left(k_{\|}^{2}+\eta k_{z}^{2}\right), \quad \text { for } \omega \ll t^{\prime} \quad(3 \mathrm{~d}) .
$$

In this $3 \mathrm{~d}$ regime, the momenta are now restricted to $k_{z} \ll \Lambda$ and $k_{\|} \ll \Lambda_{\eta}$. In both the energy ranges $\omega \ll t^{\prime}$ and $t^{\prime} \ll \omega \ll t$, the limiting forms of the dispersion, (5) and (7), respectively, are sufficient for the description of thermodynamics. These forms are independent of microscopic details, the only requirement being the existence of a dimensional crossover scale $\propto t^{\prime}$. In contrast, the precise properties of the crossover itself depend on details of the band structure, i.e., Eq. (4), and are non-universal. As we are less interested in these non-universal details, we approximate the dispersion by

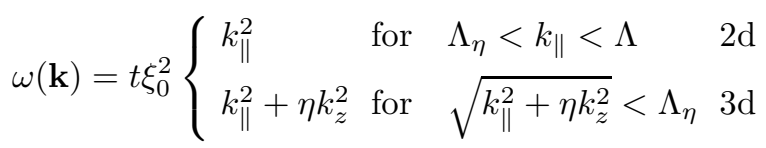

We will use the dispersion (8) for the propagator of the spin fluctuations

$$
\begin{aligned}
& \chi_{0}^{-1}\left(\mathbf{k}, i \omega_{n}\right)= \\
& \delta_{0}+\frac{\left|\omega_{n}\right|}{\Gamma_{\mathbf{k}}}+\xi_{0}^{2}\left\{\begin{array}{llll}
k_{\|}^{2} & \text { for } & \Lambda_{\eta}<k_{\|} \ll \Lambda & 2 \mathrm{~d} \\
k_{\|}^{2}+\eta k_{z}^{2} & \text { for } & \sqrt{k_{\|}^{2}+\eta k_{z}^{2}}<\Lambda_{\eta} & 3 \mathrm{~d}
\end{array}\right.
\end{aligned}
$$

Here, $\delta_{0}$ represents the bare mass of the spin fluctuations, and the dynamics of the spin fluctuations is encoded in the Landau damping term $\left|\omega_{n}\right| / \Gamma_{\mathbf{k}}$ which will be discussed in the next subsection. 
Varying $\delta_{0}$ drives the system through the QPT, which occurs at $\delta_{0}=\delta_{0, \text { cr }}$. We define the control parameter $r$ of the QPT via

$$
r=\delta_{0}-\delta_{0, \mathrm{cr}},
$$

such that $r=0$ at the QCP. To calculate observables like thermal expansion, we shall assume that the phase transition can be tuned by changing the pressure $p$, which is reflected in a pressure dependence of the bare mass $\delta_{0}(p)$.

\section{B. Landau damping}

The dynamics of the paramagnetic spin fluctuations is controlled by damping due to particle-hole excitations in the metal; this Landau damping term $\left|\omega_{n}\right| / \Gamma_{\mathbf{k}}$ dominates over the undamped dynamical term $\propto \omega_{n}^{2}$ in the propagator at low energies. The function $\Gamma_{\mathbf{k}}$ has to be obtained from an expansion of the particle-hole bubble of the host metal, for small energies and momenta close to the ordering wavevector. As the damping is different for ferro- and antiferromagnets, the different energy regimes discussed above need to be distinguished. Note that in the following we assume that the Fermi wavevector is sufficiently large for fermions to cause damping, $2 k_{F}>|\mathbf{Q}|$.

Let us start with the dimensional crossover from $2 \mathrm{~d}$ AFM to $3 \mathrm{~d}$ AFM. In both asymptotic regimes, the wavevector $\mathbf{Q}$ connects hot lines on the Fermi surface, hence the known result for the antiferromagnet, $\Gamma_{\mathbf{k}}=$ const, applies. As we are not interested in details of the crossover, we shall employ

$$
\Gamma_{\mathbf{k}}=\left\{\begin{array}{lll}
\Gamma_{2 d} \text { for } & \Lambda_{\eta}<k_{\|}<\Lambda, & 2 \mathrm{dAFM} \\
\Gamma_{3 d} \text { for } \sqrt{k_{\|}^{2}+\eta k_{z}^{2}}<\Lambda_{\eta}, & \text { 3d AFM }
\end{array}\right.
$$

The dimensional crossover from $2 \mathrm{~d} F \mathrm{FM}$ to $3 \mathrm{~d}$ AFM is somewhat more complicated. The ordering wavevector is $\mathbf{Q}=\left(0,0, Q_{z}\right)$. In the $2 \mathrm{~d}$ regime, i.e., for energies $\omega \gg t^{\prime}$, we assume that not only the spin fluctuation spectrum, but also the fermionic dispersion is $2 \mathrm{~d}$. Without dispersion in the vertical direction, we obtain for $\Gamma_{\mathbf{k}}$ the standard result for the ferromagnet, $\Gamma_{\mathbf{k}} \propto k_{\|}$. On the other hand, in the $3 \mathrm{~d}$ regime we again have $\Gamma_{\mathbf{k}}=$ const for the AFM. Hence, we approximate $\Gamma_{\mathbf{k}}$ by

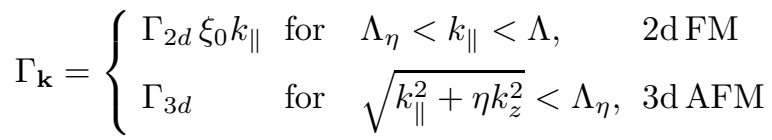

An explicit evaluation of the Landau damping for FM fluctuations in the presence of an anisotropic Fermi surface is given in App. A. The result given there, Eq. (A6), reduces to the limiting form of (12) in the $2 \mathrm{~d}$ FM regime.

In principle, the damping coefficients in the two different regimes, $\Gamma_{2 d}$ and $\Gamma_{3 d}$, differ from each other. However, as they only determine the overall energy scale, we will set them, for simplicity, equal from now on, $\Gamma=\Gamma_{2 d}=\Gamma_{3 d}$ (see also the discussion in Sec. IIE below).

\section{Correlation length}

The quartic interaction $u_{0}$ among spin fluctuations in (11) will modify its bare susceptibility (9). For our model, this modification is captured by an effective correlation length $\xi$,

$$
\begin{aligned}
& \chi^{-1}\left(\mathbf{k}, i \omega_{n}\right)= \\
& \xi^{-2}+\left\{\begin{array}{llll}
\left|\omega_{n}\right| k_{\|}^{2-z}+k_{\|}^{2} & \text { for } & \Lambda_{\eta}<k_{\|}<\Lambda & 2 \mathrm{~d} \\
\left|\omega_{n}\right|+k_{\|}^{2}+\eta k_{z}^{2} & \text { for } & \sqrt{k_{\|}^{2}+\eta k_{z}^{2}}<\Lambda_{\eta} & 3 \mathrm{~d}
\end{array}\right.
\end{aligned}
$$

where in the $2 \mathrm{~d}$ regime the dynamical exponent is either $z=2$ for AFM or $z=3$ for FM fluctuations. From now on, we will employ dimensionless units, i.e., we set effectively the length scale $\xi_{0}=1$ and the energy scale $\Gamma=1$. In addition, we use a unit volume $V=1$.

For $d+z>4$, the correlation length $\xi$ can be obtained from a self-consistent perturbation theory in $u_{0}$ :

$$
\xi^{-2}=\delta_{0}+\frac{N+2}{6} u_{0} T \sum_{\mathbf{k} \omega_{n}} \chi\left(\mathbf{k}, i \omega_{n}\right) .
$$

Only in a regime of $2 \mathrm{~d}$ antiferromagnetic fluctuations, this formula misses logarithmic corrections to the correlation length. In order to capture these, we will later have to apply the renormalization group (RG).

Substituting the sum over Matsubara frequencies by an integral over the real axis, we obtain

$$
\begin{aligned}
\xi^{-2}= & \delta_{0}+\frac{N+2}{6} u \int_{0}^{\Lambda_{\omega}} \frac{d \omega}{\pi} \operatorname{coth} \frac{\omega}{2 T} \\
& {\left[\int_{\Lambda_{\eta}}^{\Lambda} \frac{d k k}{2 \pi} \frac{\omega k^{2-z}}{\left(\xi^{-2}+k^{2}\right)^{2}+\left(\omega k^{2-z}\right)^{2}}\right.} \\
& \left.+\frac{\pi}{\Lambda_{\eta}} \int_{0}^{\Lambda_{\eta}} \frac{d k k^{2}}{2 \pi^{2}} \frac{\omega}{\left(\xi^{-2}+k^{2}\right)^{2}+\omega^{2}}\right]
\end{aligned}
$$

where $\Lambda_{\omega}$ is an additional energy cutoff, and we introduced the two-dimensional quartic coupling $u=u_{0} \Lambda / \pi$. The factor $\Lambda / \pi$ originates from the dummy momentum integration over the $z$ component in the $2 \mathrm{~d}$ regime. The contribution in the second (third) line is attributed to the spin fluctuations of effectively two (three)-dimensional character. Correspondingly, we define a $2 \mathrm{~d}(3 \mathrm{~d})$ regime in the phase diagram plane where the second (third) line in Eq. (15) dominates the correlation length. We obtain the following criterion:

$$
\begin{array}{lll}
T>\Lambda_{\eta}^{2(z-1)} & \text { or } \quad r>\Lambda_{\eta}^{2(z-1)} & 2 d \text { regime } \\
T<\Lambda_{\eta}^{2(z-1)} & \text { and } \quad r<\Lambda_{\eta}^{2(z-1)} & 3 d \text { regime }
\end{array}
$$

where $z=2$ for $2 \mathrm{~d}$ AFM and $z=3$ for $2 \mathrm{~d}$ FM spin fluctuations. 


\section{Thermodynamics}

From the susceptibility (13), we can obtain the free energy of the critical spin fluctuations

$$
F_{\mathrm{cr}}=\frac{N T}{2} \sum_{\mathbf{k}, \omega_{n}} \log \chi^{-1}\left(\mathbf{k}, i \omega_{n}\right) .
$$

It will be convenient to absorb a factor $\Lambda / \pi$ in the units of the free energy, $F \pi / \Lambda \rightarrow F$. Doing so, the free energy in our approximation takes the form

$$
\begin{aligned}
F_{\text {cr }}= & -\frac{N}{2} \int_{0}^{\Lambda_{\omega}} \frac{d \omega}{\pi} \operatorname{coth} \frac{\omega}{2 T}\left[\int_{\Lambda_{\eta}}^{\Lambda} \frac{d k k}{2 \pi} \arctan \frac{\omega k^{2-z}}{\xi^{-2}+k^{2}}\right. \\
& \left.+\frac{\pi}{\Lambda_{\eta}} \int_{0}^{\Lambda_{\eta}} \frac{d k k^{2}}{2 \pi^{2}} \arctan \frac{\omega}{\xi^{-2}+k^{2}}\right] .
\end{aligned}
$$

The integral in the first (second) line originates from the $2 \mathrm{~d}(3 \mathrm{~d})$ spin fluctuations.

From the free energy, we can compute thermodynamic properties. We will consider the specific heat, thermal expansion, Grüneisen parameter and the compressibility. The specific-heat coefficient $\gamma$ is defined as

$$
\gamma=-\frac{\partial^{2} F}{\partial T^{2}} .
$$

The thermal expansion $\alpha$ measures the change in volume as the temperature is changed,

$$
\alpha=\left.\frac{1}{V} \frac{\partial V}{\partial T}\right|_{p}=\frac{1}{V} \frac{\partial^{2} F}{\partial p \partial T}=-\left.\frac{1}{V} \frac{\partial S}{\partial p}\right|_{T} .
$$

Using a Maxwell equation, we have re-written the thermal expansion as a derivative of entropy with respect to pressure. In principle, all parameters of the model (11) might be pressure dependent. However, it has been argued ${ }^{12}$ that the most important contribution comes from the pressure dependence of the parameter multiplying the most relevant operator in the model. Close to the quantum critical point, this is the control parameter $r$ of the transition. Near a pressure-tuned quantum critical point, we can expand the control parameter around the critical pressure $p_{c}, r \approx\left(p-p_{c}\right) / p_{0}$, where $p_{0}$ is an a priori unknown pressure scale. In this case, there is a contribution to thermal expansion that measures the change of entropy upon variations of the control parameter $r$. Choosing dimensionless units, this contribution is given by

$$
\alpha_{\mathrm{cr}}=\frac{\partial F_{\mathrm{cr}}}{\partial T \partial r} .
$$

The critical Grüneisen parameter $\Gamma_{\text {cr }}$ is the ratio of critical thermal expansion and specific heat,

$$
\Gamma_{\mathrm{cr}}=\frac{\alpha_{\mathrm{cr}}}{T \gamma_{\mathrm{cr}}} .
$$

The compressibility $\kappa$ measures the change in volume as the pressure is changed with temperature held fixed,

$$
\kappa=-\left.\frac{1}{V} \frac{\partial V}{\partial p}\right|_{T}=-\frac{1}{V} \frac{\partial^{2} F}{\partial p^{2}} .
$$

The quantum critical fluctuations contribute to $\kappa$ an additive term, in the following denoted by $\kappa_{\mathrm{cr}}$. The pressure dependence of the control parameter $r$ results in the following contribution to $\kappa_{\mathrm{cr}}$ :

$$
\kappa_{\mathrm{cr}}=-\frac{\partial^{2} F_{\mathrm{cr}}}{\partial r^{2}}
$$

in dimensionless units.

\section{E. Universality?}

Before presenting actual results, it is worth asking how "universal" we can expect them to be. This question has various aspects: (i) Are the crossover functions (for a specific observable) universal in the sense that they do not depend on microscopic details - at least in a certain well-defined limit? (ii) Do all observables display the same crossover scale(s)?

For aspect (i) the answer is that full universality does not exist, as the quantum phase transitions under consideration are at or above their upper critical dimension. Therefore, even if the ultraviolet cut-off $\Lambda$ and the crossover scale $\Lambda_{\eta}$ are well separated, i.e. $\eta \ll 1$, the bare values of $\Lambda$ and the paramagnon interaction $u$ will influence the crossover functions. In particular, the interaction $u$ is at the origin of the classical dimensional crossover lines denoted as $T_{c l}$ in Figs. 1 and 2

Moreover, as discussed at length in Sec. IA the dimensional crossover itself is determined by microscopic details, i.e. the precise crossover form of the bare susceptibility. At this point, possibly existing magnetic interlayer frustration enters $\stackrel{14}{ }$ Similarly, the reference energy scales of the $2 \mathrm{~d}$ and $3 \mathrm{~d}$ regimes, $\Gamma_{2 d}$ and $\Gamma_{3 d}$, which we have taken to be equal for simplicity, may differ (by a factor of order unity), which leads to a shift of the two asymptotic regimes on the temperature axis w.r.t. each other.

For some of the observables listed above, additional care has to be taken regarding the dependence on external pressure. As the pressure dependence of all microscopic parameters is smooth, singular contributions to thermodynamics usually arise only through the pressure dependence of $r$ in the vicinity of the QCP. However, in our model, the $2 \mathrm{~d}$ regime possesses another relevant parameter, namely the anisotropy $\eta$. If the anisotropy also depends on pressure, it will give important additional contributions to thermal expansion and compressibility in the $2 \mathrm{~d}$ regime. In dimensional units, these contributions are represented by

$$
\alpha_{\mathrm{cr}}^{\eta}=\frac{\partial F_{\mathrm{cr}}}{\partial T \partial \eta}, \quad \kappa_{\mathrm{cr}}^{\eta}=-\frac{\partial^{2} F_{\mathrm{cr}}}{\partial \eta^{2}} .
$$


They can be best estimated by considering the free energy $F$ with a momentum dependence for the spinfluctuations given by Eq. (4), instead of the simplified expression for $F$, (18). In the $2 \mathrm{~d}$ regime, we obtain that the pressure dependence of the anisotropy yields contributions proportional to the ones deriving from a pressuredependent control parameter, $\alpha_{\mathrm{cr}}^{\eta} \propto \alpha_{\mathrm{cr}}$ and $\kappa_{\mathrm{cr}}^{\eta} \propto \kappa_{\mathrm{cr}}$. In the $3 \mathrm{~d}$ regime, on the other hand, $\alpha_{\mathrm{cr}}^{\eta}$ and $\kappa_{\mathrm{cr}}^{\eta}$ are suppressed by additional powers of momenta compared to $\alpha_{\mathrm{cr}}$ and $\kappa_{\mathrm{cr}}$, respectively, and are therefore negligible. Having this in mind, we omit a further discussion of these terms in the following sections.

Given all these caveats, our calculations to be presented below are nevertheless valuable, for various reasons: (a) they illustrate the general structure of the phase diagram with the quantum and classical dimensional crossover lines, (b) they show the existence of the interesting intermediate regime where $2 \mathrm{~d}$ quantum fluctuations coexist with 3d classical fluctuations, (c) they show which observables are especially sensitive to the dimensional crossover and how "broad" or "narrow" the crossover signatures are expected to be, and (d) they show where logarithmic corrections can dominate power laws.

Let us briefly comment on aspect (ii), namely whether all observables display the same crossover scale. For thermodynamics, there is a single relevant momentum crossover scale $\Lambda_{\eta}$, at least in our simple model without inter-layer frustration. The single momentum scale however translates into various temperature scales, and depending on the thermodynamic quantity of interest different crossover scales might be of importance. For the $2 \mathrm{~d}$ - 3d AFM crossover, there are two relevant temperature scales: the quantum dimensional crossover temperature $T \sim \Lambda_{\eta}^{2}$, and the classical crossover temperature $T_{c l}$, determined by the paramagnon interaction $u$. Whereas the specific-heat coefficient is insensitive to the $T_{c l}$ scale, the thermal expansion and compressibility show signatures at both scales, and their dimensional crossover appears to be broad due to the existence of the intermediate region, $\Lambda_{\eta}^{2}<T<T_{c l}$. For the 2d FM - 3d AFM crossover, we find even three temperature scales arising from the single momentum scale $\Lambda_{\eta}$ : a quantum dimensional crossover temperature $T \sim \Lambda_{\eta}^{4}$, a temperature scale $T \sim \Lambda_{\eta}^{3}$ dividing $2 \mathrm{~d}$ non-critical from $2 \mathrm{~d}$ quantum critical behavior, and a classical dimensional crossover scale $T \sim T_{c l}$. These multiple crossover temperatures result in rich thermodynamics and render the extraction of power laws especially difficult.

Transport properties - which are not subject of this paper - can be expected to display even more complicated crossover behavior. In addition to the momentum scale $\Lambda_{\eta}$, there are characteristic length scales for transport scattering processes. For coupled chains, it has been argued 21 that the interplay of those length and time scales can render transport fully three-dimensional even in a regime where the thermodynamic behavior is $1 \mathrm{~d}$. Hence, thermodynamic and transport crossovers do not have to coincide in general.

\section{DIMENSIONAL CROSSOVER: 2D ANTIFERROMAGNET TO 3D ANTIFERROMAGNET}

For materials consisting of antiferromagnetic planes, which itself are weakly coupled in the third direction, a scenario of a crossover from $2 \mathrm{~d}$ antiferromagnetism to $3 \mathrm{~d}$ antiferromagnetism is plausible. As mentioned in the introduction, such a scenario might be realized in the heavy-fermion metal $\mathrm{CeCu}_{6-x} \mathrm{Au}_{x}$. In the following, we present an analysis of this crossover within the framework of the LGW model whose applicability to $\mathrm{CeCu}_{6-x} \mathrm{Au}_{x}$, however, has been questioned, ${ }^{9,10}$

\section{A. Renormalization group}

The Hertz model (11) for AFM fluctuations in $2 \mathrm{~d}$ is at its upper critical dimension, $d+z=d_{c}^{+}=4$. Consequently, there are important logarithmic corrections in perturbation theory that have to be summed, e.g., with the help of the renormalization group (RG). The $R G$ equations for the running mass $\delta(b)$ and quartic coupling $u(b)$ in the $2 \mathrm{~d}$ AFM regime read: $\underline{1,2,4}$

$$
\begin{aligned}
& \frac{\partial \delta}{\partial \log b}=2 \delta-\frac{N+2}{12 \pi^{2}} u \delta, \\
& \frac{\partial u}{\partial \log b}=-\frac{N+8}{12 \pi^{2}} u^{2} .
\end{aligned}
$$

Here, we have introduced the RG scale $b$, and the RG flow corresponds to a reduction of the momentum-space cutoff, $\Lambda \rightarrow \Lambda / b$. The one-loop correction to $\delta$, i.e., the tadpole diagram, can be expanded in a power series in $\delta$ : The constant term is finite and non-universal (i.e. cutoffdependent), whereas the linear term is universal. The latter linear term is written as second term in Eq. (26a); the constant term will be absorbed in the initial value of $\delta$. Hence, the flow starts at $b=1$ with the initial conditions $\delta(b=1)=r_{2 d}$ (that differs from $\delta_{0}$ by the non-universal Hartree shift) and $u(b=1)=u=u_{0} \Lambda / \pi$, being the effective $2 \mathrm{~d}$ quartic coupling. Note that the prefactors in (26) have been evaluated at $T=0$, as their temperature dependence is subleading.

Integrating the $\mathrm{RG}$ equation for the quartic coupling we obtain

$$
u(b)=\frac{12 \pi^{2}}{N+8} \frac{1}{\log \frac{b \Lambda}{\Lambda}},
$$

where we have introduced the momentum scale $\bar{\Lambda}$, which depends on the bare quartic coupling constant $u$,

$$
\bar{\Lambda} \equiv \Lambda e^{\frac{12 \pi^{2}}{(N+8) u}} .
$$


Using the running quartic coupling, the solution for the control parameter is easily obtained. Substituting $\delta(b)=$ $r(b) b^{2}$, we get

$$
r(b)=\frac{r_{2 d}}{\left(\log \left[b \frac{\bar{\Lambda}}{\Lambda}\right]\right)^{\frac{N+2}{N+8}}}
$$

As we will see later, the parameter $r_{2 d}$ differs from the control parameter, $r \equiv r_{3 d}$, of the quantum phase transition by corrections of order $\Lambda_{\eta}^{2}$.

\section{B. Correlation length}

The logarithmic scale dependence of the coupling and the control parameter has to be in calculating the correlation length. Hence, Eq. (15) is replaced by

$$
\begin{aligned}
& \xi^{-2}=r\left(\Lambda / \Lambda^{*}\right) \\
& +\frac{N+2}{6} u\left(\Lambda / \Lambda^{*}\right) \int_{0}^{\infty} \frac{d \omega}{\pi} \int_{\Lambda_{\eta}}^{\Lambda} \frac{d k k}{2 \pi} \frac{\omega\left(\operatorname{coth} \frac{\omega}{2 T}-1\right)}{\left(\xi^{-2}+k^{2}\right)^{2}+\omega^{2}} \\
& +\frac{N+2}{6} \frac{\pi u^{*}}{\Lambda_{\eta}} \int_{0}^{\Lambda_{\omega}} \frac{d \omega}{\pi} \int_{0}^{\Lambda_{\eta}} \frac{d k k^{2}}{2 \pi^{2}} \frac{\omega \operatorname{coth} \frac{\omega}{2 T}}{\left(\xi^{-2}+k^{2}\right)^{2}+\omega^{2}}
\end{aligned}
$$

The abbreviations $\Lambda^{*}$ and $u^{*}$ are defined through

$$
\Lambda^{*}=\max \left\{\xi^{-1}, \Lambda_{\eta}\right\}
$$

and

$$
u^{*}=u\left(\Lambda / \Lambda_{\eta}\right)=\frac{12 \pi^{2}}{N+8} \frac{1}{\log \frac{\bar{\Lambda}}{\Lambda_{\eta}}},
$$

the latter being the quartic coupling at the crossover scale $\Lambda_{\eta}$. Eq. (30) can be understood as follows: The first line accounts for the $2 \mathrm{~d}$ quantum fluctuations at $T=0$ which have been re-summed in $r(b)$ using the RG, see Eq. (29). Finite-temperature corrections to this $2 \mathrm{~d}$ result can be treated perturbatively ${ }^{22}$ and are in the second line. The third line, finally, is attributed to the $3 \mathrm{~d}$ fluctuations.

In the following, the limiting behavior of the correlation length is discussed in detail.

\section{Correlation length in the $2 d$ regime, $T \gg \Lambda_{\eta}^{2}$ or $r_{2 d} \gg \Lambda_{\eta}^{2}$}

In the $2 \mathrm{~d}$ regime as defined in Eq. (16), we can neglect the last line in the expression (30) as it yields only a small correction. We further distinguish two sub-regimes.

Fermi-liquid regime, $T \ll r_{2 d}$ : In the Fermi-liquid regime, the correlation length is determined by the solution of the RG equation for the tuning parameter

$$
\xi^{-2}=\frac{r_{2 d}}{\left(\log \frac{\bar{\Lambda}}{\sqrt{r_{2 d}}}\right)^{\frac{N+2}{N+8}} .}
$$

The logarithmic dependence can be understood as an incipient correction to the mean-field value of the correlation length exponent $\nu_{\mathrm{MF}}=1 / 2$.

Quantum critical regime, $T \gg r_{2 d}$ : The limiting behavior of the remaining integral is given by the small $\omega$ limit of the integrand, such that

$$
\xi^{-2}=r\left(\Lambda / \Lambda^{*}\right)+\frac{\pi}{2} \frac{N+2}{N+8} T \frac{\log \frac{T}{\Lambda^{* 2}}}{\log \frac{\bar{\Lambda}}{\Lambda^{*}}} .
$$

Hence, in the quantum critical regime, the correlation length is determined by temperature, $\xi^{-2} \sim T$, up to logarithmic corrections. The logarithms are either cutoff by the correlation length itself or by the dimensional crossover scale $\Lambda_{\eta}$. In the limit $\xi^{-2} \gg \Lambda_{\eta}^{2}$, the correlation length is asymptotically given by

$\xi^{-2}=r\left(\frac{\Lambda}{\sqrt{T}}\right)+\frac{\pi}{2} \frac{N+2}{N+8} T \frac{\log \log \frac{\bar{\Lambda}}{\sqrt{T}}}{\log \frac{\bar{\Lambda}}{\sqrt{T}}}, \quad$ for $\xi^{-2} \gg \Lambda_{\eta}^{2}$.

The $\log \log$ dependence in the numerator can be attributed to the $2 \mathrm{~d}$ classical fluctuations, i.e, the $2 \mathrm{~d}$ like Matsubara zero mode, within the quantum critical regime. [Depending on whether the first or the second term dominates in (35), one can further distinguish two sub-regimes within the regime denoted as the renormalized $2 \mathrm{~d}$ classical regime in Fig. 1].] When the correlation length exceeds the crossover scale, i.e. $\xi^{-2} \ll \Lambda_{\eta}^{2}$, the logarithms are now cut-off by $\Lambda_{\eta}$,

$$
\xi^{-2}=r\left(\Lambda / \Lambda_{\eta}\right)+\frac{\pi}{2} \frac{N+2}{N+8} T \frac{\log \frac{T}{\Lambda_{\eta}^{2}}}{\log \frac{\bar{\Lambda}}{\Lambda_{\eta}}}, \quad \text { for } \quad \xi^{-2} \ll \Lambda_{\eta}^{2} .
$$

The crossover at the scale $\xi^{-2} \sim \Lambda_{\eta}^{2}$ is associated with the advocated dimensional crossover for the classical critical fluctuations, where the Matsubara zero mode changes its character from $2 \mathrm{~d}$ to $3 \mathrm{~d}$. At criticality, $r_{2 d}=0$, this occurs at a temperature

$$
\left.T_{c l}\right|_{r_{2 d}=0} \sim \Lambda_{\eta}^{2} \frac{\log \frac{\bar{\Lambda}}{\Lambda_{\eta}}}{\log \log \frac{\bar{\Lambda}}{\Lambda_{\eta}}} .
$$

This classical dimensional crossover temperature, $T_{c l}$, is logarithmically enhanced as compared to the quantum dimensional crossover temperature, $T \sim \Lambda_{\eta}^{2}$, see Fig. 1]

The classical dimensional crossover occuring at $T_{c l}$ becomes non-perturbative when the line $T_{c l}(r)$ enters the classical Ginzburg region. Generally, our perturbative treatment breaks down sufficiently close to the classical transition at the Ginzburg temperature $T_{G}$, where the classical Ginzburg parameter is of order one, $\mathcal{G} \sim \mathcal{O}(1)$. For 3d classical fluctuations, the Ginzburg parameter is given by $\mathcal{G}=U \xi$, where the classical quartic coupling is $U=\pi u^{*} T / \Lambda_{\eta} \sim T /\left(\Lambda_{\eta} \log \frac{\bar{\Lambda}}{\Lambda_{\eta}}\right)$. Upon increasing temperature, the crossover line $T_{c l}(r)$ approaches $T_{G}(r)$ and 
enters the Ginzburg region at a temperature $T_{x}$ where $T_{G}$ and $T_{c l}$ coincide, see Fig. 1.

$$
T_{x} \sim \Lambda_{\eta}^{2} \log \frac{\bar{\Lambda}}{\Lambda_{\eta}} .
$$

2. Correlation length in the $3 d$ regime, $T \ll \Lambda_{\eta}^{2}$ and

$$
r_{3 d} \ll \Lambda_{\eta}^{2}
$$

In the $3 \mathrm{~d}$ regime, we can instead neglect the second line in (30). We again distinguish between two sub-regimes.

Fermi-liquid regime, $T \ll r_{3 d}$ : In the Fermi-liquid regime, we can set $T=0$ in (30) in order to obtain the leading estimate for the correlation length,

$$
\xi^{-2}=r_{3 d} \equiv \frac{r_{2 d}}{\left(\log \frac{\bar{\Lambda}}{\Lambda_{\eta}}\right)^{\frac{N+2}{N+8}}}+\mathcal{C} \frac{\Lambda_{\eta}^{2}}{\log \frac{\bar{\Lambda}}{\Lambda_{\eta}}},
$$

where $\mathcal{C}$ is a non-universal constant that depends on the chosen cut-off structure. The control parameter $r \equiv r_{3 d}$ obtains a shift from the contribution of the parameter regime where the spin fluctuations have developed their three-dimensional character; the consequences of this shift are discussed in Sec. IIIB3, The temperature correction neglected in (39) is of Fermi-liquid type $\sim T^{2}$.

Quantum critical regime, $T \gg r_{3 d}$ : In the quantum critical regime, the temperature corrections dominate the correlation length. Evaluating the leading behavior we obtain

$$
\xi^{-2}=r_{3 d}+\left(\frac{\pi}{2}\right)^{3 / 2} \zeta(3 / 2) \frac{N+2}{N+8} \frac{T^{3 / 2}}{\Lambda_{\eta} \log \frac{\bar{\Lambda}}{\Lambda_{\eta}}},
$$

where we used the explicit expression for $u^{*}$, Eq. (32). The quantum critical regime can again be further subdivided into regimes where either of the above two terms dominates the correlation length.

\section{Phase boundary}

The position of the phase boundary, $T_{c}(r)$, in the $(T, r)$ plane can be estimated by setting the correlation length to infinity. In the quantum $2 \mathrm{~d}$ regime for temperatures lower than the crossing temperature $T<T_{x}$, Eq. (38), the classical dimensional crossover from $2 \mathrm{~d}$ to $3 \mathrm{~d}$ is perturbative as it is located outside the classical Ginzburg region. Here, we can use expression (36) for the correlation length to obtain

$$
r_{2 d}=-\frac{\pi}{2} \frac{N+2}{N+8} \frac{T_{c} \log \frac{T_{c}}{\Lambda_{\eta}}}{\left(\log \frac{\bar{\Lambda}}{\Lambda_{\eta}}\right)^{1-\frac{N+2}{N+8}}} .
$$

For larger $T>T_{x}$, the logarithmic corrections to the critical temperature $T_{c}$ will differ from Eq. (41) due to the non-perturbative character of the classical dimensional crossover. Neglecting the logarithmic corrections, the phase boundary varies linearly with temperature, $T_{c} \propto r_{2 d}$, in the $2 \mathrm{~d}$ regime.

In the $3 \mathrm{~d}$ regime, on the other hand, we use expression (40) and get

$$
r_{3 d}=-\left(\frac{\pi}{2}\right)^{3 / 2} \zeta(3 / 2) \frac{N+2}{N+8} \frac{T_{c}^{3 / 2}}{\Lambda_{\eta} \log \frac{\bar{\Lambda}}{\Lambda_{\eta}}} .
$$

The different behavior of the phase boundary in the $2 \mathrm{~d}$ and $3 \mathrm{~d}$ regimes, (41) and (42), implies that an extrapolation of the phase boundary from the $2 \mathrm{~d}$ regime leads to an incorrect position of the quantum critical point. The $3 \mathrm{~d}$ spin-fluctuations shift the quantum critical point slightly towards the ordered phase. The quantum critical point is not at $r_{2 d}=0$, but rather at $r_{3 d}=0$, leading to a difference between the extrapolated and the actual position of the QCP,

$$
\Delta r=\left.r_{3 d}\right|_{r_{2 d}=0} \sim \frac{\Lambda_{\eta}^{2}}{\log \frac{\bar{\Lambda}}{\Lambda_{\eta}}} .
$$

The position of the extrapolated QCP, $\Delta r$, see Fig. 目, is, however, located close to or even within the $3 \mathrm{~d}$ pocket of the phase diagram.

\section{Thermodynamics}

We turn to a discussion of the thermodynamic quantities specified in Sec. IID They can be obtained with the help of expression (18) for the free energy, with the correlation length given by the self-consistent Eq. (30). A numerical solution for the specific heat, thermal expansion, and compressibility in the quantum critical regime is presented in Fig. 5. A detailed discussion of their asymptotic behavior is given below.

1. Thermodynamics in the $2 d$ regime, $T \gg \Lambda_{\eta}^{2}$ or $r_{2 d} \gg \Lambda_{\eta}^{2}$

Within the $2 \mathrm{~d}$ regime, the thermodynamics is dominated by the first line in the expression for the free energy Eq. (18). We again distinguish between two sub-regimes.

Fermi-liquid regime, $T \ll r_{2 d}$ : The evaluation of the specific heat and thermal expansion is straightforward

$$
\gamma_{\mathrm{cr}}=\frac{N}{6} \log \frac{\Lambda}{\sqrt{r_{2 d}}}, \quad \alpha_{\mathrm{cr}}=\frac{N}{12} \frac{T}{r_{2 d}} .
$$

The resulting Grüneisen parameter reads

$$
\Gamma_{\mathrm{cr}}=\frac{1}{2} \frac{1}{r_{2 d} \log \frac{\Lambda}{\sqrt{r_{2 d}}}} .
$$

The evaluation of the compressibility is more involved. Here, we have to take into account the effective momentum dependence of the correlation length, $\xi_{k}^{-2}=r(\Lambda / k)$, 


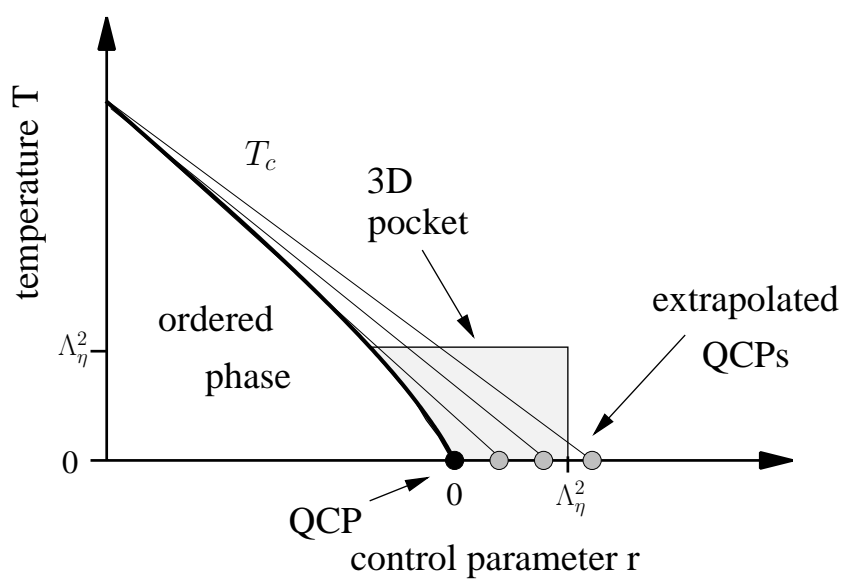

FIG. 4: Locating the QCP based on experimental data requires an extrapolation of the finite- $T$ phase boundary to $T=0$. Such an extrapolation procedure is prone to a systematic error if a dimensional crossover at lower temperatures takes place. The extrapolation of the phase boundary (thick line) from the $2 \mathrm{~d}$ regime towards $T=0$ yields estimates of the position of the QCP that are shifted towards the non-ordered phase by an amount $\Delta r$. The different thin lines and the associated extrapolated QCPs illustrate that the extrapolation itself is ambiguous as the $2 \mathrm{~d}$ phase boundary is not strictly linear in $T$ but has logarithmic corrections. For the $2 \mathrm{~d}-3 \mathrm{~d}$ AFM crossover, both the size of the $3 \mathrm{~d}$ pocket and the shift $\Delta r$, Eq. (43), are of order $\Lambda_{\eta}^{2}$. For the 2d FM - 3d AFM crossover of Sec. IV the size of the $3 \mathrm{~d}$ pocket is instead given by $\Lambda_{\eta}^{4}$, while the shift $\Delta r \propto \Lambda_{\eta}^{2}$, Eq. (70). Consequently, the extrapolated QCP is outside the $3 \mathrm{~d}$ pocket in this case.

see Eq. (29), in the expression for the free energy (18) that arises after RG improvement of perturbation theory. We obtain

$$
\kappa_{\mathrm{cr}}=\frac{N}{4 \pi^{2}} \int_{\Lambda^{*}} \frac{d k}{k}\left(\frac{\partial r(\Lambda / k)}{\partial r_{2 d}}\right)^{2} .
$$

The asymptotic behavior originates from the lower limit of the momentum integral that has to be cutoff either by the crossover scale or the correlation length itself, $\Lambda^{*}=$ $\max \left\{\xi^{-1}, \Lambda_{\eta}\right\}$. In the $2 \mathrm{~d}$ regime we thus obtain for the asymptotic behavior: 23,24

$$
\kappa_{\mathrm{cr}}=\frac{N}{4 \pi^{2}} \frac{1}{1-2 \frac{N+2}{N+8}}\left(\log \frac{\bar{\Lambda}}{\sqrt{r_{2 d}}}\right)^{1-2 \frac{N+2}{N+8}} .
$$

Note that, for $N<4$, Eq. (47) predicts a correction to the compressibility that diverges upon approaching the quantum critical point, $r_{2 d} \rightarrow 0$. This divergence is only cutoff upon entering the $3 \mathrm{~d}$ regime.

A diverging electronic compressibility has interesting consequences. In particular, a coupling of the electronic system to lattice degrees of freedom can render the coupled system unstable, resulting in a first-order transition driven by quantum critical AFM fluctuations $\stackrel{25}{\underline{2}}$ Such a fluctuation-driven first-order transition may occur in the present case for a sufficiently large $2 \mathrm{~d}$ regime.
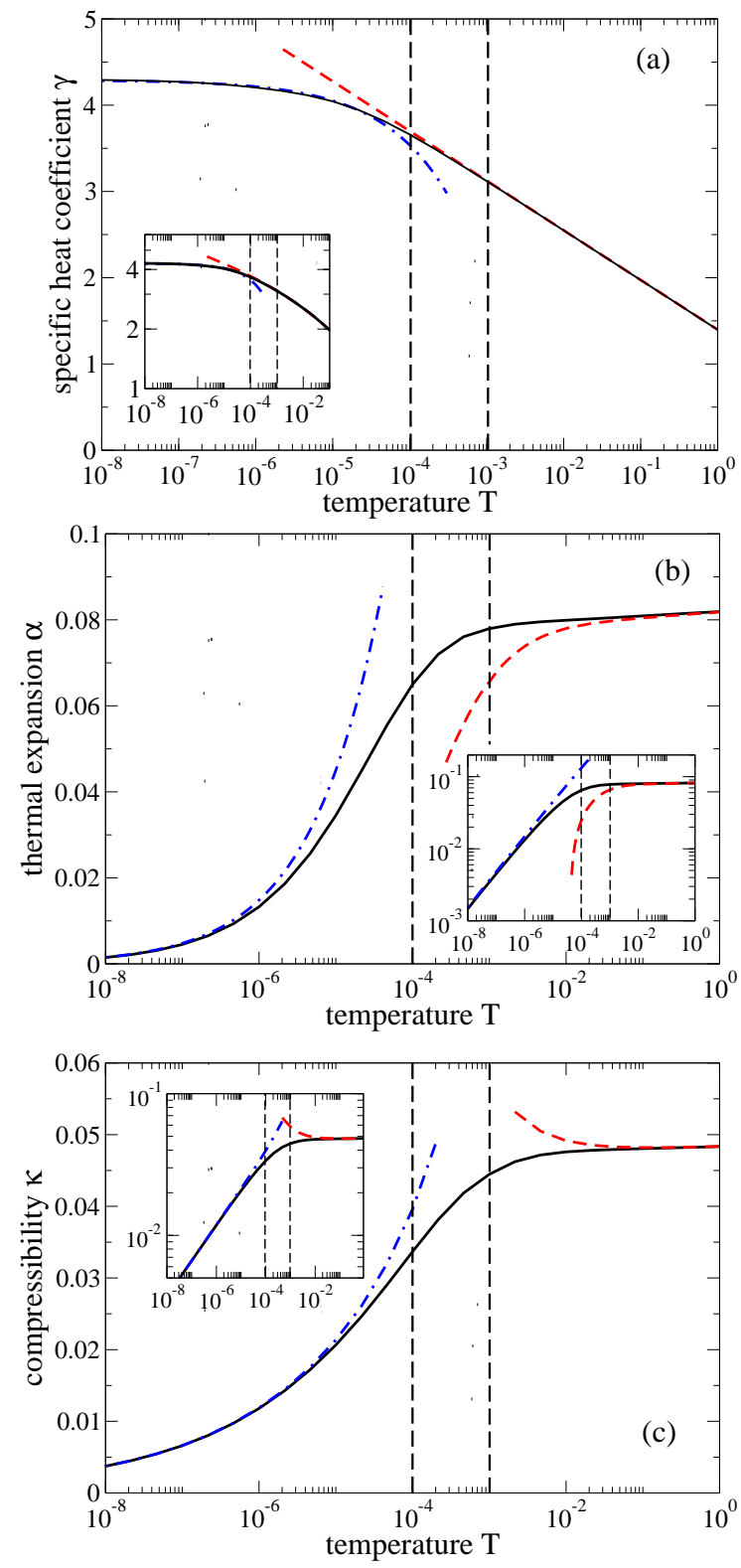

FIG. 5: (color online) Crossover behavior of thermodynamics for $r=0$, i.e., in the quantum critical regime of the 2d AFM - 3d AFM crossover scenario. The vertical dashed line at higher $T \sim T_{c l}$ indicates the classical and the one at lower $T \sim \Lambda_{\eta}^{2}$ the quantum dimensional crossover temperature. The insets show the same data on a double-logarithmic scale. The chosen parameters are $\Lambda_{\omega}=\Lambda=20, \Lambda_{\eta}=0.01$, $u=1$ and $N=3$. Panel (a): Specific-heat coefficient $\gamma$. Also shown are the asymptotic behaviors given by Eq. (48) (red/dashed) for the high-temperature 2d regime and Eq. (58) (blue/dash-dot) for the low-temperature $3 \mathrm{~d}$ regime. Panel (b): Thermal expansion $\alpha$. The asymptotic behaviors are given by Eq. (49) (red/dashed) and by Eq. (59) (blue/dashdot). Panel (c): Compressibility $\kappa$. The asymptotics are in Eq. (52) (red/dashed) and Eq. (60) (blue/dash-dot). Note the very weak increase with $T$ in the high-temperature $2 \mathrm{~d}$ regime. 
Quantum critical regime, $T \gg r_{2 d}$ : In the $2 \mathrm{~d}$ quantum critical regime, the specific heat depends logarithmically on temperature,

$$
\gamma_{\mathrm{cr}}=\frac{N}{6} \log \frac{\Lambda}{\sqrt{T}}
$$

For the thermal expansion we obtain

$$
\alpha_{\mathrm{cr}}=\frac{N}{8 \pi} \frac{\partial \xi^{-2}}{\partial r_{2 d}}\left(\log \frac{T}{\xi^{-2}+\Lambda_{\eta}^{2}}+\text { const }\right) .
$$

This leads to slightly different asymptotic behavior within the $2 \mathrm{~d}$ and $3 \mathrm{~d}$ classical regime, see Fig. 1,

$$
\alpha_{\mathrm{cr}} \sim\left\{\begin{array}{lll}
\left(\log \frac{\bar{\Lambda}}{\sqrt{T}}\right)^{-\frac{N+2}{N+8}} \log \log \frac{\bar{\Lambda}}{\sqrt{T}} & \text { if } & T \gg T_{c l} \\
\left(\log \frac{\bar{\Lambda}}{\Lambda_{\eta}}\right)^{-\frac{N+2}{N+8}} \log \frac{T}{\Lambda_{\eta}^{2}} & \text { if } & T \ll T_{c l}
\end{array} .\right.
$$

For $T \gg T_{c l}$, we obtain for the Grüneisen parameter the temperature dependence

$$
\Gamma_{\mathrm{cr}} \sim \frac{\log \log \frac{\bar{\Lambda}}{\sqrt{T}}}{T \log \frac{\Lambda}{\sqrt{T}}\left(\log \frac{\bar{\Lambda}}{\sqrt{T}}\right)^{\frac{N+2}{N+8}}} .
$$

The critical part of the compressibility is dominated by the Matsubara zero mode. Its form also differs in the classical $2 \mathrm{~d}$ and $3 \mathrm{~d}$ regime,

$$
\kappa_{\mathrm{cr}}=\left(\frac{\partial \xi^{-2}}{\partial r_{2 d}}\right)^{2} \times\left\{\begin{array}{lll}
\frac{N}{8 \pi} \frac{T}{\xi^{-2}} & \text { if } & T \gg T_{c l} \\
\frac{N}{16} \frac{T}{\Lambda_{\eta} \xi^{-1}} & \text { if } & T \ll T_{c l}
\end{array}\right.
$$

Using the expressions for the correlation length (35) and (36), we obtain the asymptotic behavior at criticality $r_{2 d}=0$,

$$
\kappa_{\mathrm{cr}} \sim\left\{\begin{array}{l}
\left(\log \frac{\bar{\Lambda}}{\sqrt{T}}\right)^{-2 \frac{N+2}{N+8}} \frac{\log \frac{\bar{\Lambda}}{\sqrt{T}}}{\log \log \frac{\Lambda}{\sqrt{T}}} \quad \text { if } \quad T \gg T_{c l} \\
\frac{\sqrt{T}}{\Lambda_{\eta}}\left(\log \frac{\bar{\Lambda}}{\Lambda_{\eta}}\right)^{-2 \frac{N+2}{N+8}} \sqrt{\frac{\log \frac{\bar{\Lambda}}{\Lambda_{\eta}}}{\log \frac{T}{\Lambda_{\eta}^{2}}}} \text { if } \quad T \ll T_{c l}
\end{array}\right.
$$

The asymptotics of thermal expansion and compressibility in the logarithmically small intermediate regime $\Lambda_{\eta}^{2}<T<T_{c l}$ are not shown in Fig. 5.

\section{Thermodynamics in the $3 d$ regime, $T \ll \Lambda_{\eta}^{2}$ and $r_{3 d} \ll \Lambda_{\eta}^{2}$}

In the $3 \mathrm{~d}$ regime, the critical contributions to thermodynamics are coming from the second line in Eq. (18).

Fermi-liquid regime, $T \ll r_{3 d}$ : Here, the specific heat has the form

$$
\gamma=\frac{N}{6} \log \frac{\Lambda}{\Lambda_{\eta}}+\gamma_{\mathrm{cr}}, \quad \gamma_{\mathrm{cr}}=-\frac{N \pi}{12} \frac{\sqrt{r_{3 d}}}{\Lambda_{\eta}} .
$$

The critical part, $\gamma_{\mathrm{cr}}$, that depends on the control parameter, $r_{3 \mathrm{~d}}$, leads here only to a small correction to the background contribution that originates from the $2 \mathrm{~d}$ fluctuations. The thermal expansion reads

$$
\alpha_{\mathrm{cr}}=\frac{N \pi}{24} \frac{T}{\Lambda_{\eta} \sqrt{r_{3 d}}} .
$$

We omit here and in the following multiplicative factors that are powers of $\partial \xi^{-2} / \partial r_{2 d}$, that includes the logarithmic normalization of the $2 \mathrm{~d}$ control parameter, see (39). In the Fermi-liquid regime the critical Grüneisen ratio, $\Gamma_{\mathrm{cr}}=\alpha_{\mathrm{cr}} /\left(T \gamma_{\mathrm{cr}}\right)$, reads

$$
\Gamma_{\mathrm{cr}}=-\frac{1}{2} \frac{1}{r_{3 d}}
$$

As explained in Ref. 12, in the Fermi-liquid regime scaling predicts a universal critical Grüneisen ratio in the sense that the proportionality factor in the relation $\Gamma_{\text {cr }} \propto 1 / r$ is just determined by critical exponents. The prefactor $-1 / 2$ in Eq. (56) is in agreement with this scaling prediction. For the compressibility we get

$$
\kappa_{\mathrm{cr}}=-\frac{N}{8 \pi} \frac{\sqrt{r_{3 d}}}{\Lambda_{\eta}} .
$$

Quantum critical regime, $T \gg r_{3 d}$ : Again, the critical part of the specific heat is only sub-leading (54) now with

$$
\gamma_{\mathrm{cr}}=-\frac{15 \zeta(5 / 2) N}{\sqrt{2 \pi} 32} \frac{\sqrt{T}}{\Lambda_{\eta}}
$$

The thermal expansion is given by

$$
\alpha_{\mathrm{cr}}=N \frac{3 \zeta(3 / 2)}{\sqrt{2 \pi} 16} \frac{\sqrt{T}}{\Lambda_{\eta}} .
$$

The thermal expansion behaves as $\sqrt{T}$ in the $3 \mathrm{~d}$ regime. The sub-leading correction to (59) vanishes as $T^{3 / 4}$, with a two-fold origin. First, there is a contribution due to the next-to-leading term in the expansion of the second derivative of the free energy $\partial^{2} F_{\mathrm{cr}} / \partial T \partial \xi^{-2}$ that is of order $\xi^{-1} / \Lambda_{\eta}$. Second, and more importantly, there is a $T$-dependent correction originating from the derivative $\partial \xi^{-2} / \partial r_{3 \mathrm{~d}}$ that contributes to $\alpha$ a term of order $T^{3 / 2} / \xi^{-1} \Lambda_{\eta}^{2}$. Both contributions originate from the $T$ dependence of the correlation length, induced by the bosonic interaction $u{ }^{27}$ The critical Grüneisen parameter deriving from (58) and (59) obeys scaling, $\Gamma_{\mathrm{cr}} \sim 1 / T$.

The compressibility is dominated by the Matsubara zero mode,

$$
\kappa_{\mathrm{cr}}=\frac{N}{16} \frac{T \xi}{\Lambda_{\eta}} \sim\left\{\begin{array}{lll}
\sqrt{\frac{\log \frac{\bar{\Lambda}}{\Lambda_{\eta}}}{\Lambda_{\eta}}} T^{1 / 4} & \text { if } & r_{3 d} \ll \frac{T^{3 / 2}}{\Lambda_{\eta} \log \frac{\Lambda}{\Lambda_{\eta}}} \\
\frac{T}{\sqrt{r_{3 d}} \Lambda_{\eta}} & \text { if } & r_{3 d} \gg \frac{T^{3 / 2}}{\Lambda_{\eta} \log \frac{\Lambda}{\Lambda_{\eta}}}
\end{array}\right.
$$


where we used the expression (40) for the correlation length. There exist two sub-regimes where the compressibility varies either linearly with $T$ or, very close to the quantum critical point, as $T^{1 / 4}$ (Ref. 26). Note that the critical contribution to the compressibility, $\kappa_{\mathrm{cr}}$, changes sign upon crossing over from the Fermi liquid (57) to the quantum critical regime (60).

\section{DIMENSIONAL CROSSOVER: 2D FERROMAGNET TO 3D ANTIFERROMAGNET}

We now turn to the dimensional crossover from a $2 \mathrm{~d}$ ferromagnet to a $3 \mathrm{~d}$ antiferromagnet, describing the situation of weakly AFM coupled ferromagnetic planes, where the $3 \mathrm{~d}$ ordered state corresponds to so-called Atype antiferromagnetism.

The key difference to the crossover in Sec. III is related to the form of Landau damping and, as a consequence, the different dynamical exponents $z=3$ and $z=2$ in the $2 \mathrm{~d}$ and $3 \mathrm{~d}$ regimes, respectively. The combination of low spatial dimensionality, $d=2$, and large dynamical exponent, $z=3$, results in strong thermodynamic contributions of the $2 \mathrm{~d}$ FM spin fluctuations. Even below the temperature $T \lesssim \Lambda_{\eta}^{3}$, thermally excited $2 \mathrm{~d}$ fluctuations lead to a large non-critical Fermi liquid background that dominates over the critical 3d AFM fluctuations in a wide parameter range, see Fig. 2. This leads to a peculiar situation at criticality, $r=0$ : the $2 \mathrm{~d}$ and $3 \mathrm{~d}$ quantum critical regions are separated by a temperature regime $\Lambda_{\eta}^{4}<T<\Lambda_{\eta}^{3}$ where non-critical Fermi liquid behavior prevails.

Other differences between the present FM - AFM crossover and the AFM - AFM crossover of Sec. III are: For the FM - AFM crossover, the uniform susceptibility changes from critical to non-critical behavior, see Sec. IVC. Finally, the effective dimensionality is always larger than the upper critical dimension, $d+z>4$. Therefore, we can use directly Eqs. (15) and (18) for the correlation length and free energy, respectively, without the need of a RG improvement. This simplifies the analysis considerably.

\section{A. Correlation length}

In the analysis of the asymptotic behavior of the correlation length, we again distinguish between the $2 \mathrm{~d}$ and the $3 \mathrm{~d}$ regime, where $\xi$ is dominated by the first and second integral in (15), respectively.

\section{Correlation length in the $2 d$ regime, $T \gg \Lambda_{\eta}^{4}$ or $r_{2 d} \gg \Lambda_{\eta}^{4}$}

Fermi-liquid regime, $T \ll r_{2 d}^{3 / 2}$ and $r_{2 d} \gg \Lambda_{\eta}^{2}$ : Evaluating the correlation length in the $2 \mathrm{~d}$ Fermi-liquid regime we obtain

$$
\xi^{-2}=r_{2 d}+\frac{\pi(N+2)}{144} u \frac{T^{2}}{r_{2 d}^{3 / 2}}
$$

where $r_{2 d}$ differs from the bare mass $\delta_{0}$ by a constant cutoff-dependent shift. Here, the temperature-dependent part is of the Fermi-liquid form and subleading.

Quantum critical regime, $T \gg r^{3 / 2}$ and $T \gg \Lambda_{\eta}^{3}$ : In the quantum critical regime, on the other hand, temperature dominates the correlation length

$$
\xi^{-2}=r_{2 d}+\frac{N+2}{24 \pi} u T \log \frac{1}{\Lambda^{* 2} T^{-2 / 3}}
$$

with $\Lambda^{*}=\max \left\{\xi^{-1}, \Lambda_{\eta}\right\}$ as above, Eq. (31). The $2 \mathrm{~d}$ quantum critical regime can be subdivided into three regimes depending on how $\xi^{-2}$ compares with $\Lambda_{\eta}^{2}$, and whether the first or the temperature-dependent second term dominates. At $\xi^{-2} \sim \Lambda_{\eta}^{2}$, a dimensional crossover associated with the classical critical fluctuations takes place. At criticality, $r \approx r_{2 d}=0$, this crossover occurs at a temperature of order

$$
\left.T_{c l}\right|_{r=0} \sim \frac{\Lambda_{\eta}^{2}}{u \log \frac{1}{\Lambda_{\eta}}} .
$$

At this classical dimensional crossover, the logarithmic increase of 62 with increasing correlation length $\xi$ is cutoff. This allows, in particular, a solution for the phase boundary, see below. However, at a higher temperature

$$
T_{x} \sim \frac{\Lambda_{\eta}^{2}}{u}
$$

$T_{c l}(r)$ enters the Ginzburg regime and the classical dimensional crossover and, as a consequence, the expression for the phase boundary becomes non-perturbative.

Non-critical Fermi liquid regime, $T \ll \Lambda_{\eta}^{3}$ and $r_{2 d} \ll$ $\Lambda_{\eta}^{2}$ : The $2 \mathrm{~d}$ critical behavior crosses over into a $2 \mathrm{~d}$ noncritical Fermi liquid behavior below a temperature $T \sim$ $\Lambda_{\eta}^{3}$ or, as a function of the control parameter at $r \sim \Lambda_{\eta}^{2}$, see the shaded lines in Fig. 2. In this intermediate regime, the correlation length has the form,

$$
\xi^{-2}=r_{2 d}+\mathcal{C}_{1} u \frac{T^{2}}{\Lambda_{\eta}^{3}},
$$

where the numerical factor $\mathcal{C}_{1}$ is non-universal, i.e., depends on our modeling of the dimensional crossover. It turns out that this $2 \mathrm{~d}$ Fermi-liquid background still dominates thermodynamics down to a temperature and control parameter scale $\Lambda_{\eta}^{4}$ where finally the $3 \mathrm{~d}$ critical behavior takes over.

$$
\begin{aligned}
& \text { 2. Correlation length in the } 3 d \text { regime, } T \ll \Lambda_{\eta}^{4} \text { and } \\
& \qquad r_{3 d} \ll \Lambda_{\eta}^{4}
\end{aligned}
$$

Fermi-liquid regime, $T \ll r_{3 d}$ : The contribution from the $3 \mathrm{~d}$ fluctuations shift the value for the control param- 
eter

$$
\xi^{-2}=r_{3 d} \equiv r_{2 d}+\mathcal{C}_{2} u \Lambda_{\eta}^{2}+\frac{\pi(N+2)}{144} u \frac{T^{2}}{\Lambda_{\eta} r_{3 d}^{1 / 2}}
$$

where $\mathcal{C}_{2}$ is a constant dependent on the employed cutoff structure. The temperature dependence is again of Fermi liquid type. Comparing the temperature corrections to the correlation length in the various Fermi liquid regimes, one obtains the two control parameter crossover scales, $\Lambda_{\eta}^{4}$ and $\Lambda_{\eta}^{2}$, that are shown in Fig. 2,

Quantum critical regime, $T \gg r_{3 d}$ : In the quantum critical regime we get

$$
\xi^{-2}=r_{3 d}+(N+2) \frac{\zeta(3 / 2)}{24 \sqrt{2 \pi}} u \frac{T^{3 / 2}}{\Lambda_{\eta}} .
$$

A comparison of this temperature dependence with the one of Eq. 65) yields the quantum dimensional crossover temperature, $T \sim \Lambda_{\eta}^{4}$.

\section{Phase boundary}

In the limit of vanishing correlation length, Eq. (62) yields for $T_{c}<T_{x}$, see Eq. (64), the following expression for the phase boundary within the quantum critical $2 \mathrm{~d}$ regime

$$
r_{2 d}=-\frac{N+2}{24 \pi} u T_{c} \log \frac{T_{c}^{2 / 3}}{\Lambda_{\eta}^{2}} .
$$

It varies (up to logarithmic corrections) linearly with $T$. In the intermediate non-critical Fermi liquid regime, the phase boundary behaves as $r_{2 d} \sim-u T_{c}^{2} / \Lambda_{\eta}^{3}$, before crossing over into the $3 \mathrm{~d}$ regime. There, we use expression 676 to obtain

$$
r_{3 d}=-(N+2) \frac{\zeta(3 / 2)}{24 \sqrt{2 \pi}} u \frac{T_{c}^{3 / 2}}{\Lambda_{\eta}} .
$$

Similar to Sec. IIIB3 the extrapolation of the phase boundary within the $2 \mathrm{~d}$ regime towards $T=0$ leads to an error in the estimate for position of the quantum critical point of

$$
\Delta r=\left.r_{3 d}\right|_{r_{2 d}=0} \sim u \Lambda_{\eta}^{2}
$$

\section{B. Thermodynamics}

Thermodynamic quantities follow from the expression (18) for the free energy. A numerical solution for the specific heat, thermal expansion and compressibility in the quantum critical regime is shown in Fig. 6. In the following, an analysis of the asymptotic behavior is presented.
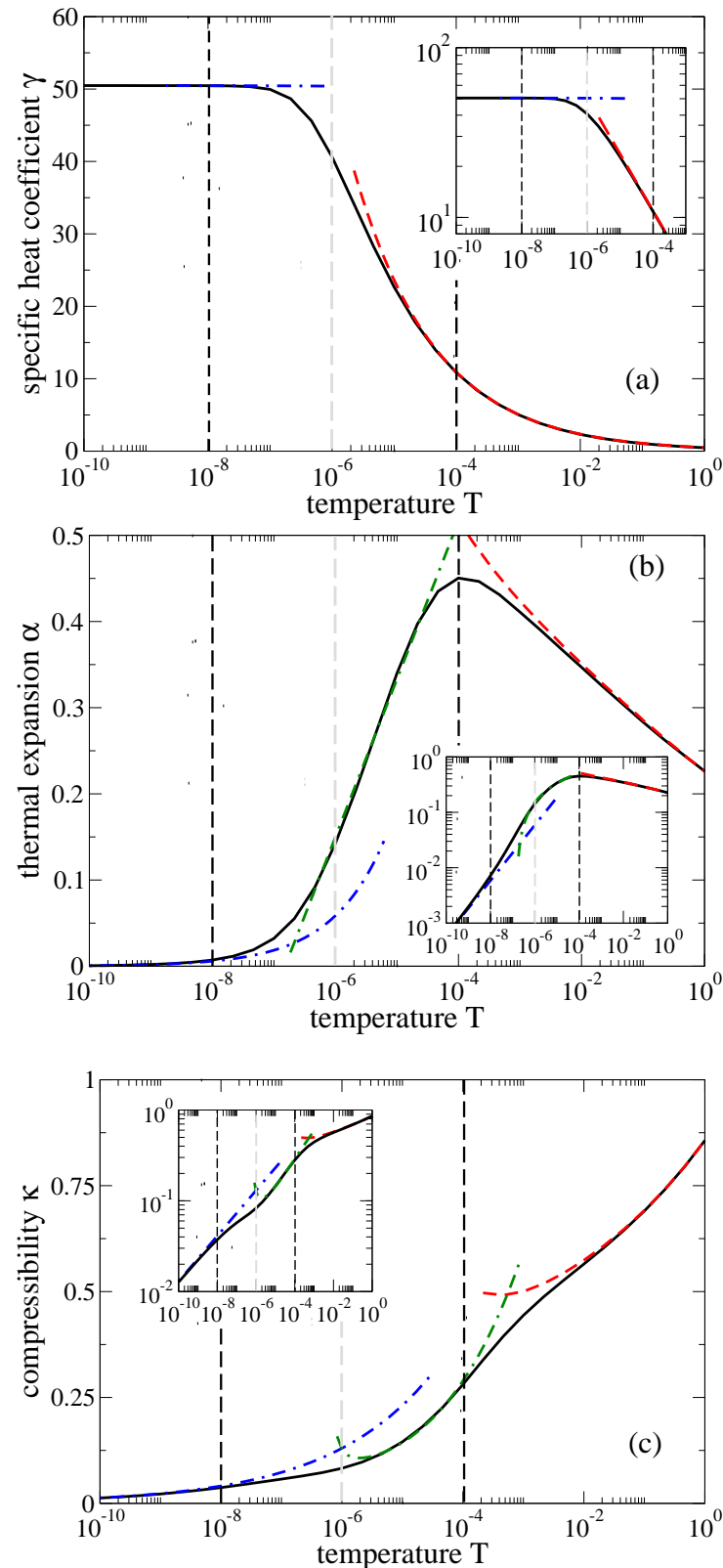

FIG. 6: (color online) Crossover behavior as in Fig. 5, but now for the 2d FM - 3d AFM case. The vertical dashed lines denote the three crossover scales $T_{c l}>\Lambda_{\eta}^{3}>\Lambda_{\eta}^{4}$, see Fig. 2. $T_{c l}$ denotes the classical dimensional crossover where $\xi \sim 1 / \Lambda_{\eta} ; T \sim \Lambda_{\eta}^{3}$ separates the 2 d critical regime from the $2 \mathrm{~d}$ non-critical Fermi-liquid regime; and below $T \sim \Lambda_{\eta}^{4} 3 \mathrm{~d}$ quantum critical behavior sets in. The chosen parameters are $\Lambda_{\omega}=\Lambda=20, \Lambda_{\eta}=0.01, u=1$ and $N=3$. Panel (a): Specific-heat coefficient $\gamma$, with the asymptotics given by Eq. (74) (red/dashed) for the high-temperature $2 \mathrm{~d}$ regime and by Eq. (58) (blue/dash-dot) for the low-temperature $3 \mathrm{~d}$ regime. Panel (b): Thermal expansion $\alpha$, showing a maximum at the classical dimensional crossover. The asymptotics at high (red/dashed) and intermediate $T$ (green/dashdash-dot) are given by (76), and at low $T$ (blue/dash-dot) by Eq. (59) The inset shows that the asymptotic $\sqrt{T}$ behavior only sets in for $T<\Lambda_{\eta}^{4}=10^{-8}$. Panel (c): Compressibility $\kappa$, with the asymptotics in Eq. (78) (red/dashed and green/dash-dash-dot) and Eq. (60) (blue/dash-dot) showing the $T^{1 / 4}$ behavior that again only sets in for $T<\Lambda_{\eta}^{4}$. 
1. Thermodynamics in the $2 d$ regime, $T \gg \Lambda_{\eta}^{4}$ or $r_{2 d} \gg \Lambda_{\eta}^{4}$

Fermi-liquid regime, $T \ll r_{2 d}^{3 / 2}$ and $r_{2 d} \gg \Lambda_{\eta}^{2}$ : In the 2d Fermi-liquid regime, evaluating the leading behavior is straightforward. For the specific-heat coefficient and thermal expansion we obtain

$$
\gamma_{\mathrm{cr}}=\frac{N \pi}{12} \frac{1}{\sqrt{r_{2 d}}}, \quad \alpha_{\mathrm{cr}}=\frac{N \pi}{24} \frac{T}{r_{2 d}^{3 / 2}} .
$$

This gives a universal Grüneisen parameter ${ }^{12}$

$$
\Gamma_{\mathrm{cr}}=\frac{1}{2} \frac{1}{r_{2 d}} .
$$

For the critical part of the compressibility we find

$$
\kappa_{\mathrm{cr}}=-\frac{N}{8 \pi} \sqrt{r_{2 d}}
$$

Quantum critical regime, $T \gg r_{2 d}^{3 / 2}$ and $T \gg \Lambda_{\eta}^{3}$ : In the $2 \mathrm{~d}$ quantum critical regime, the specific heat is given by

$$
\gamma_{\mathrm{cr}}=\frac{N}{6 \pi} \Gamma\left(\frac{8}{3}\right) \zeta\left(\frac{5}{3}\right) T^{-1 / 3} .
$$

The thermal expansion depends logarithmically on temperature

$$
\alpha_{\mathrm{cr}}=\frac{N}{8 \pi} \log \frac{T^{2 / 3}}{\max \left\{\xi^{-2}, \Lambda_{\eta}^{2}\right\}}+\text { const }
$$

with the correlation length given in Eq. (62). Using its explicit form, it becomes clear that the thermal expansion has a maximum at the dimensional crossover, $\xi^{-1} \sim \Lambda_{\eta}$, that occurs at a temperature $T_{c l}$ and is associated with the classical Matsubara zero mode:

$$
\alpha_{\mathrm{cr}} \sim\left\{\begin{array}{lll}
\log \frac{1}{u T^{1 / 3}} & \text { if } & T \gg T_{c l} \\
\log \frac{T^{2 / 3}}{\Lambda_{\eta}^{2}}+\text { const. } & \text { if } & T \ll T_{c l}
\end{array} .\right.
$$

In the quantum critical $2 \mathrm{~d}$ regime, the Grüneisen parameter shows the asymptotic behavior

$$
\Gamma_{\mathrm{cr}} \sim \frac{1}{T^{2 / 3}}
$$

where we omitted logarithmic corrections that depend on the effective dimensionality of the classical fluctuations, see Eq. (76).

Similarly, the compressibility also exhibits an additional dimensional crossover at $T_{c l}$ as it is determined by the classical fluctuations

$$
\kappa_{\mathrm{cr}}=\left\{\begin{array}{lll}
\frac{N}{8 \pi} \frac{T}{\xi^{-2}} & \text { if } & T \gg T_{c l} \\
\frac{N}{16} \frac{T}{\Lambda_{\eta} \xi^{-1}} & \text { if } & T \ll T_{c l}
\end{array}\right.
$$

Using Eq. (62) for the correlation length we obtain the asymptotic behavior for the compressibility at criticality, $r \approx r_{2 d}=0$,

$$
\kappa_{\mathrm{cr}} \sim\left\{\begin{array}{lll}
\frac{1}{u \log \frac{1}{T}} \cdot & \text { if } & T \gg T_{c l} \\
\frac{\sqrt{T}}{\Lambda_{\eta} \sqrt{u}} & \text { if } & T \ll T_{c l}
\end{array} .\right.
$$

Note that $\kappa$ depends singularly on the dangerously irrelevant interaction $u$.

The results (76) and (779) show that not only the $2 \mathrm{~d}$ quantum critical regime above $T_{c l}$, but also the intermediate $2 \mathrm{~d}$ quantum $3 \mathrm{~d}$ classical critical regime below $T_{c l}$ displays a well-defined universal asymptotic behavior.

Non-critical Fermi liquid regime, $T \ll \Lambda_{\eta}^{3}$ and $r_{2 d} \ll$ $\Lambda_{\eta}^{2}$ : In the intermediate non-critical regime, thermodynamic quantities display a Fermi-liquid form:

$$
\gamma=\frac{1}{2 \Lambda_{\eta}}-\mathcal{C}_{3} \frac{T^{2}}{\Lambda_{\eta}^{7}}, \quad \alpha=\mathcal{C}_{4} \frac{T}{\Lambda_{\eta}^{3}}
$$

with constants $\mathcal{C}_{3}$ and $\mathcal{C}_{4}$ being non-universal. The compressibility is determined by $3 \mathrm{~d}$ classical fluctuations,

$$
\kappa_{\mathrm{cr}}=\frac{N}{16} \frac{T}{\Lambda_{\eta} \xi^{-1}},
$$

where the correlation length $\xi$ has the form (65).

$$
\begin{aligned}
& \text { 2. Thermodynamics in the } 3 d \text { regime, } T \ll \Lambda_{\eta}^{4} \text { and } \\
& \qquad r_{3 d} \ll \Lambda_{\eta}^{4}
\end{aligned}
$$

The thermodynamics in the $3 \mathrm{~d}$ regime resembles the one discussed in Sec. III C. The background contribution for the specific heat coefficient in Eq. (54) is, however, now determined by the $2 \mathrm{~d}$ FM fluctuations, $\gamma-\gamma_{\mathrm{cr}}=\frac{1}{2 \Lambda_{\eta}}$.

\section{Uniform susceptibility}

A particular property of the $2 \mathrm{~d} F M-3 \mathrm{~d}$ AFM crossover is that the susceptibility, measuring the response to a uniformly applied magnetic field, is critical within the $2 \mathrm{~d}$ regime, whereas it becomes non-critical at the dimensional crossover. We consider the static limit and study its temperature dependence

$$
\chi_{\mathrm{u}}(T) \equiv \chi\left(T, \Omega_{n}=0, k_{\|}=0, k_{z}=Q_{z}\right)=\frac{1}{\xi^{-2}+\xi_{Q}^{-2}} .
$$

As before, the wavevector $\mathbf{k}$ is measured relative to the (3d) ordering wavevector $\mathbf{Q} . \xi_{Q}^{-2}$ is a constant proportional to the hopping $t^{\prime}$ between the $2 \mathrm{~d}$ planes, $\xi_{Q}^{-2} \propto$ $t^{\prime}\left[1-\cos \left(Q_{z} a\right)\right]$. From the discussion in Sec. II it follows that $\xi_{Q}^{-2} \sim \Lambda_{\eta}^{2}$. The temperature behavior is fully accounted for by the $T$ dependence of the correlation length 


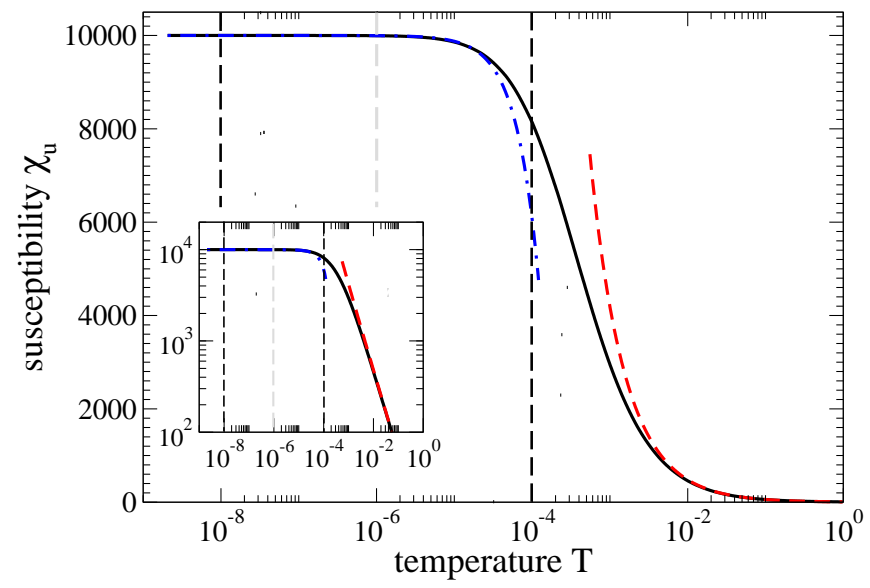

FIG. 7: Behavior of the uniform susceptibility $\chi_{\mathrm{u}}$ for $r=0$, i.e., in the quantum critical regime of the $2 \mathrm{~d}$ FM - 3d AFM crossover scenario. The chosen parameters are $\xi_{Q}^{-2}=0.0001$, $\Lambda_{\omega}=\Lambda=20, \Lambda_{\eta}=0.01, u=1$ and $N=3$. The vertical dashed lines represent the crossover scales $T_{Q}=\xi_{Q}^{-2} \sim \Lambda_{\eta}^{2}<$ $\Lambda_{\eta}^{3}<\Lambda_{\eta}^{4}$. The dashed/red line is the $2 \mathrm{~d}$ high- $T$ asymptote (83), and the dashed-dot/blue line is the $3 \mathrm{~d}$ low- $T$ asymptote (84).

$\xi$. In the quantum critical $2 \mathrm{~d}$ regime, i.e., at high temperature, we can neglect the constant contribution $\xi_{Q}^{-2}$, and we obtain with the help of (62) for the asymptotic behavior

$$
\chi_{\mathrm{u}}(T) \sim \frac{1}{T \log \frac{1}{T}}
$$

This divergence is cut off below a scale $T_{Q}=\xi_{Q}^{-2} \sim \Lambda_{\eta}^{2}$, i.e. essentially at the classical dimensional crossover line. For $T \ll T_{Q}$, the constant contribution from the $t^{\prime}$ dispersion dominates, $\chi_{\mathrm{u}}=$ const. In the $3 \mathrm{~d}$ quantum critical regime, the temperature dependence of the correlation length (67) leads to the $T$-dependent correction $\chi_{\mathrm{u}}=$ const $+\delta \chi_{\mathrm{u}}(T)$,

$$
\delta \chi_{\mathrm{u}} \sim-T^{3 / 2},
$$

in agreement with the results of Ref. 28. The result of a numerically evaluation of the uniform susceptibility (82) is shown in Fig. 7. The lower two crossover temperatures leave essentially no trace in $\chi_{\mathrm{u}}$, as the regimes only differ in the small thermal correction to the large $\chi_{\mathrm{u}}(T=0)$.

\section{APPLICATION TO HEAVY-FERMION METALS}

Our results are of potential relevance to layered nearly magnetic metals where indications for two-dimensional criticality have been found. In this section, we discuss the cases of the heavy-fermion materials $\mathrm{CeCu}_{6-x} \mathrm{Au}_{x}$, $\mathrm{YbRh}_{2} \mathrm{Si}_{2}$, and $\mathrm{CeCoIn}_{5}$. While $\mathrm{CeCoIn}_{5}$ possesses a layered lattice structure and should naturally display a dimensional crossover, for $\mathrm{CeCu}_{6-x} \mathrm{Au}_{x}$ and $\mathrm{YbRh}_{2} \mathrm{Si}_{2}$ there is only empirical evidence (discussed below) for quasi-2d critical behavior, with no obvious microscopic reason. We note that, in all three cases, ingredients beyond the LGW spin fluctuation theory may be important for a full understanding of the critical behavior.

We refrain from discussing strongly correlated transition-metal compounds with layered structure, such as high-temperature superconducting cuprates. In these materials, a standard LGW approach alone cannot be expected to capture the relevant physics due to the proximity to the half-filled Mott insulator.

\section{A. $\mathbf{C e C u}_{6-x} \mathbf{A u}_{x}$}

$\mathrm{CeCu}_{6}$ is a paramagnetic heavy Fermi liquid, which can be driven into an antiferromagnetic metallic phase by $\mathrm{Au}$ substitution. ${ }^{1,29}$ In $\mathrm{CeCu}_{6-x} \mathrm{Au}_{x}$, the quantum critical point is located at $x_{c} \approx 0.1$. For $x>x_{c}$, the $\mathrm{AF}$ order is known to be $3 \mathrm{~d}$, which implies non-vanishing magnetic couplings in all spatial directions. In contrast, neutron scattering in quantum-critical $\mathrm{CeCu}_{6-x} \mathrm{Au}_{x}$ has provided direct evidence for a quasi- $2 \mathrm{~d}$ antiferromagnetic fluctuation spectrum. $\underline{6}$ This suggests that a dimensional crossover occurs near the quantum critical point, which, however, has not been experimentally identified to date. Let us therefore apply our results from Sec. [II in order to search for experimentally measurable consequences of a dimensional crossover from 2d AFM to 3d AFM.

First, there is the location of the QCP itself. Experimentally, the phase boundary appears to be linear, $T_{\mathrm{N}} \propto(x-0.1)$, in other words, the data points marking the finite-temperature phase transition can be linearly extrapolated to a putative QCP at $x_{c}=0.1$. From the consideration in Sec. III it is clear that the asymptotic behavior of the phase boundary should be $T_{\mathrm{N}} \propto\left(x-x_{c}\right)^{2 / 3}$, which suggests that the true $x_{c}>0.1$. Hence, samples with $x=0.1$ may not be located at the quantum critical composition. As a consequence, the system would be located outside the $3 \mathrm{~d}$ pocket of the phase diagram in Fig. 4, this could then explain why no signatures of $3 \mathrm{~d}$ spin fluctuation were found in the neutron scattering experiment of Ref. 6. Unfortunately, concrete predictions are problematic due to the presence of logarithmic corrections in the $d=z=2$ theory for the 2d AFM: In fact, in the $2 \mathrm{~d}$ regime the phase boundary should follow $T_{\mathrm{N}} \log T_{\mathrm{N}} \propto\left(x-x_{c}\right)$, Eq. (41) - this makes a linear extrapolation ambiguous. Experimentally, such logarithmic corrections are difficult to extract.

Second, the dimensional crossover should be manifest in thermodynamics. Whereas the crossover signatures in the specific heat are weak, the thermal expansion and the compressibility show a pronounced step-like behavior in the quantum critical regime of the $2 d-3 d$ AFM crossover, see Fig. 5. The latter two are therefore more appropriate to detect a dimensional crossover in the critical spin-fluctuation spectrum. 
Thus, we propose to search for the dimensional crossover in $\mathrm{CeCu}_{6-x} \mathrm{Au}_{x}$ by (i) detecting the change in behavior of the thermal expansion at criticality and (ii) by looking for deviation of the phase boundary from linear behavior by employing pressure tuning of, e.g., an $x=0.2$ sample. From the available data, the dimensional crossover temperature is likely below $100 \mathrm{mK}$. We also note that the presence of quenched chemical disorder may modify the behavior near criticality at very low temperatures.

\section{B. $\mathbf{Y b R h}_{2} \mathbf{S i}_{2}$}

The heavy-fermion material $\mathrm{YbRh}_{2} \mathrm{Si}_{2}$ displays a phase transition at $70 \mathrm{mK} ?^{7}$ The low-temperature ordered phase is believed to be antiferromagnetic, although confirming neutron scattering data are not available to date. The ordering temperature can be suppressed by applying a small field, resulting in a field-driven QCP at $B=0.06$ $\mathrm{T}$ in the $a b$ plane and $0.66 \mathrm{~T}$ along the $c$ axis. The ordering temperature can also be suppressed by Ge doping: $\mathrm{YbRh}_{2}\left(\mathrm{Si}_{1-x} \mathrm{Ge}_{x}\right)_{2}$ with $x=5 \%$ seems to order at only $20 \mathrm{mK}$ in zero field. ${ }^{31}$ The critical properties of $\mathrm{YbRh}_{2} \mathrm{Si}_{2}$ are inconsistent with the predictions of LGW theory for 3d AFM spin fluctuations. As for $\mathrm{CeCu}_{6-x} \mathrm{Au}_{x}$, it has been speculated that the Kondo effect breaks down at quantum criticality - this idea received support from Hall effect measurements which indicate a pronounced change in the low-temperature Hall coefficient across the critical field 32

Remarkably, $\mathrm{YbRh}_{2} \mathrm{Si}_{2}$ appears to be almost ferromagnetic. This is particularly striking for $\mathrm{YbRh}_{2}\left(\mathrm{Si}_{1-x} \mathrm{Ge}_{x}\right)_{2}$ where the uniform susceptibility follows $\chi_{\mathrm{u}}(T) \propto T^{-0.6}$ above $0.3 \mathrm{~K} . \underline{\underline{8}} \mathrm{In}$ addition, the unexpected observation of an ESR signal below the Kondo temperature ${ }^{33}$ has been related to strong ferromagnetic correlations. 34

It is therefore worth discussing which properties of $\mathrm{YbRh}_{2} \mathrm{Si}_{2}$ appear consistent with the scenario of a crossover from $2 \mathrm{~d}$ FM to $3 \mathrm{~d}$ AFM, as would arise in a system of ferromagnetic layers with weak antiferromagnetic inter-layer coupling.

Interestingly, thermodynamic measurements are partially consistent with $2 \mathrm{~d}$ FM criticality, but below $0.3 \mathrm{~K}$. The specific heat follows $C(T) / T \propto T^{-0.3}$ below $0.3 \mathrm{~K}, 31$ and the Grüneisen ratio diverges as $\Gamma(T) \propto T^{-0.7}$ below $0.6 \mathrm{~K}$ (Ref. 35) - these two exponents are close to the values $-1 / 3$ and $-2 / 3$ expected for $2 \mathrm{~d}$ FM fluctuations. In addition, the temperature-field scaling observed in $\mathrm{YbRh}_{2}\left(\mathrm{Si}_{1-x} \mathrm{Ge}_{x}\right)_{2}$ with $x=5 \%$ (Ref. 36) is in accord with ferromagnetic criticality, provided that one interprets $\left(B-B_{c}\right)$ (where $B_{c}=0.027 \mathrm{~T}$ is tiny) as the field conjugate to the order parameter. However, other observations in $\mathrm{YbRh}_{2} \mathrm{Si}_{2}$ appear inconsistent with this idea of $2 \mathrm{~d}$ FM criticality: $\stackrel{37}{ }$ for example, the fractional exponent observed in the $T$ dependence of the uniform susceptibility $\chi_{\mathrm{u}}(T)$ cannot be easily explained with this scenario.

In summary, the physics of $\mathrm{YbRh}_{2} \mathrm{Si}_{2}$ cannot be explained in a straightforward manner in terms of nearcritical 2d FM fluctuations (which turn to 3d antiferromagnetism at lowest temperatures) alone. However, the experiments, showing critical ferromagnetic fluctuations which are cutoff only at very low temperatures, hint that a dimensional crossover of the type considered here may be important. Further investigations of $\mathrm{YbRh}_{2} \mathrm{Si}_{2}$ samples with Ir or Co doping 38 will shed more light on the role of the various crossover scales in this interesting material.

\section{C. $\mathrm{CeCoIn}_{5}$}

The compounds $\mathrm{CeMIn}_{5}(\mathrm{M}=\mathrm{Co}, \mathrm{Rh}$,Ir, also dubbed 115-compounds), unify a variety of fascinating heavyfermion phenomena: $\mathrm{CeCoIn}_{5}$ and $\mathrm{CeIrIn}_{5}$ are (likely unconventional) superconductors with $T_{\mathrm{c}}=2.3 \mathrm{~K}$ and $0.4 \mathrm{~K}$, respectively, whereas $\mathrm{CeRhIn}_{5}$ is an antiferromagnetic metal with $T_{\mathrm{N}} \approx 3.6 \mathrm{~K}$. Transitions between these ordered phases may be tuned using pressure, chemical substitution, or magnetic field. In contrast to most other heavy-fermion materials, $\mathrm{CeMIn}_{5}$ is quasi twodimensional, i.e., its lattice structure consists of weakly coupled layers. Consequently, a dimensional crossover scenario should naturally apply.

A particularly interesting transition occurs in $\mathrm{CeCoIn}_{5}$ upon application of a magnetic field 39 Superconductivity survives up to a critical field of $H_{c 2} \approx 4.95 \mathrm{~T}$. Normalstate properties near $H_{c 2}$ are suggestive of quantum critical behavior: both specific heat and resistivity display non-Fermi liquid temperature dependencies at $H_{c 2}$, and the $A$ coefficient of the resistivity diverges upon approaching $H_{c 2}$ from above. These features have been interpreted as signatures of an antiferromagnetic quantum critical point at (or close to) $H_{c 2}$, with the ordered phase for $H<H_{c 2}$ being suppressed by the onset of superconductivity. By applying hydrostatic pressure, the two phenomena - superconducting $H_{c 2}$ transition and apparent magnetic quantum criticality - can be separated, i.e. $H_{c 2}$ decreases faster than the quantum critical field, suggesting that the two phenomena are not related. $\underline{40}$

Recently, thermodynamic properties of $\mathrm{CeCoIn}_{5}$ near $H_{c 2}$ have been studied in more detail $\stackrel{41}{4}$ At $H=5 \mathrm{~T}$, signatures of a crossover at $T^{*} \approx 0.3 \mathrm{~K}$ between two different singular behaviors were identified. This is best visible in the thermal expansion, which was found to follow $\alpha(T) / T \propto 1 / T$ in the temperature range $0.5 \mathrm{~K}<T<$ $6 \mathrm{~K}$, whereas $\alpha(T) / T$ is consistent with $1 / \sqrt{T}$ for $0.1 \mathrm{~K}$ $<T<0.3 \mathrm{~K}$. The thermodynamic data below $T^{*} \approx 0.3 \mathrm{~K}$ have been argued to be consistent with the predictions of the LGW theory for $3 \mathrm{~d}$ AF spin fluctuations. (Note that the scale $T^{*}$, below which $3 \mathrm{~d}$ LGW behavior is seen, is shifted up to $1.4 \mathrm{~K}$ in doped $\mathrm{CeCoIn}_{5-x} \mathrm{Sn}_{x}$.) For $T>T^{*}$, the thermal expansion in $\mathrm{CeCoIn}_{5}$ seems consistent with $2 \mathrm{~d}$ AF spin fluctuations of LGW type. How- 
ever, the authors of Ref. 41 have argued that the behavior of the Grüneisen parameter, being is somewhat reminiscent to that of $\mathrm{YbRh}_{2} \mathrm{Si}_{2}$, instead suggests nonLGW criticality (which nevertheless may arise from $2 \mathrm{~d}$ critical magnetism).

Taken together, the data indicate a crossover in critical behavior at $T^{*} \approx 0.3 \mathrm{~K}$ - this is also supported by resistivity measurements which show $\rho(T)-\rho(0) \propto T^{3 / 2}$ below $T=0.2 \mathrm{~K}$ at $H=5 \mathrm{~T},{ }^{42}$ consistent with $3 \mathrm{~d}$ LGW behavior. Although the nature of the critical behavior above $T^{*}$ is not fully understood - the presence of multiple crossover scales 42 complicates the analysis of the data - the interpretation of $T^{*}$ as a dimensional crossover scale is suggestive. The role of $\mathrm{Sn}$ doping in shifting this crossover scale is unclear at present; in a situation with geometric frustration of inter-plane magnetism one might speculate that disorder partially relieves frustration. It would be worthwhile to investigate the magnetic excitations, e.g., of $\mathrm{AF}$ ordered variants of $\mathrm{CeMIn}_{5}$ by neutron scattering, in order to determine the magnetic bandwidths in the directions parallel and perpendicular to the CeIn planes.

\section{CONCLUSION}

We have studied the dimensional crossover of magnetic fluctuations in nearly quantum critical metals. Motivated by experiments on heavy-fermion systems, we have concentrated on the crossover from $2 \mathrm{~d}$ FM or AFM fluctuations at elevated energies to $3 \mathrm{~d}$ AFM fluctuations at low energies. Applying the standard Landau-GinzburgWilson approach, we have obtained relevant crossover energy scales as well as crossover functions describing thermodynamic observables.

The anisotropy in the spin-fluctuation spectrum leads to a dimensional crossover from $2 \mathrm{~d}$ to $3 \mathrm{~d}$ upon approaching criticality. We have found two types of dimensional crossover scales. Upon reducing temperature at criticality, there is a first dimensional crossover associated with the classical fluctuations and, at a lower temperature, a second crossover where the quantum fluctuations change their character from $2 \mathrm{~d}$ to $3 \mathrm{~d}$, see Figs. 1 and 2 . In particular, there is an extended intermediate temperature regime where $2 \mathrm{~d}$ quantum fluctuations coexist with $3 \mathrm{~d}$ classical fluctuations resulting in new power laws. For the 2d FM - 3d AFM crossover, there exists even a further sub-regime, where non-critical Fermi liquid behavior intervenes between the critical $2 \mathrm{~d}$ and $3 \mathrm{~d}$ regimes. We have found that the thermal expansion and the compressibility are well suited to detect a dimensional crossover of critical magnetic fluctuations. However, the existence of several dimensional crossover scales makes the experimental identification of asymptotic power laws particularly difficult.
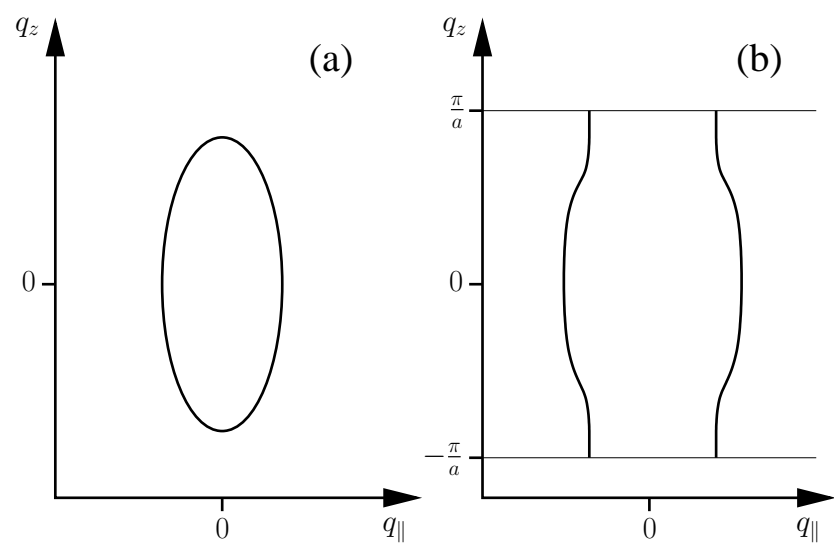

FIG. 8: Sketch of a cut through a closed (a) and open (b) 3d anisotropic Fermi surface cylindrically symmetric with respect to the $q_{z}$-axis; $q_{z}$ is the out-of-plane, and $q_{\|}$is the in-plane momentum.

\section{Acknowledgments}

We thank S. Florens, P. Gegenwart, and P. Wölfle for discussion and collaboration on related work. This research was supported by the DFG through the SFB 608, the Research Unit FG 960 "Quantum Phase Transitions", and grant FR 2627/1-1, and by the NSF through grant DMR-0757145.

\section{APPENDIX A: LINDHARD FUNCTION FOR A FERMI SURFACE WITH CYLINDRICAL SYMMETRY}

We evaluate the Landau damping of ferromagnetic fluctuations deriving from the Lindhard function for an anisotropic Fermi surface with cylindrical symmetry. The Lindhard function at $T=0$ is given by

$$
\Pi\left(k, i \omega_{n}\right)=-\int \frac{d \epsilon}{2 \pi} \sum_{q} \frac{1}{i \epsilon-\varepsilon_{q}} \frac{1}{i \epsilon+i \omega_{n}-\varepsilon_{q+k}} .
$$

In the following, we distinguish between a closed and an open anisotropic Fermi surface.

\section{Closed Fermi surface}

Here, we consider a closed anisotropic Fermi surface as, e.g., depicted in Fig. 8h. Introducing the density of states and integrating over $\epsilon$, the dynamic part of the polarization can be expressed as an integral over the (closed) $3 \mathrm{~d}$ Fermi surface

$$
\Pi_{\mathrm{dyn}}\left(k, i \omega_{n}\right)=-i \omega_{n} \int \frac{d \hat{q}}{4 \pi} \frac{\nu_{\hat{q}}}{i \omega_{n}-\mathbf{v}_{\hat{q}} \mathbf{k}},
$$

For an anisotropic Fermi surface, the density of states $\nu_{\hat{q}}$ and the Fermi velocity $\mathbf{v}_{\hat{q}}$ depend on the orientation of 
the fermionic wavevector, $\hat{q}$. We model the anisotropy of the Fermi surface with an in-plane Fermi velocity $v_{F}$ that differs from its z-component $\eta_{F} v_{F}$. A large anisotropy with a quasi two-dimensional Fermi surface is obtained in the limit of small $\eta_{F}$. So we get

$$
\begin{aligned}
\Pi_{\mathrm{dyn}}\left(k, i \omega_{n}\right) & =-\frac{1}{4 \pi} i \omega_{n} \int_{0}^{\pi} d \theta \int_{0}^{2 \pi} d \phi \\
& \times \frac{\sin \theta \nu\left(\cos ^{2} \theta\right)}{i \omega_{n}-v_{F}\left(k_{\|} \sin \theta \cos \phi+\eta_{F} k_{z} \cos \theta\right)} .
\end{aligned}
$$

We further assumed that the density of states only depends on $\cos ^{2} \theta$ with the azimuthal angle $\theta$ of the Fermi momentum. In the limit of small $\omega_{n}$, this simplifies to

$$
\Pi_{\mathrm{dyn}}\left(k, i \omega_{n}\right)=-\frac{\pi}{2}\langle\nu\rangle \frac{\left|\omega_{n}\right|}{v_{F} \sqrt{k_{\|}^{2}+\left(\eta_{F} k_{z}\right)^{2}}}
$$

where $\langle\nu\rangle$ is an angular average of the density of states that smoothly depends on momenta

$$
\langle\nu\rangle=\frac{2}{\pi} \int_{0}^{1} \frac{d x}{\sqrt{1-x^{2}}} \nu\left(\frac{k_{\|}^{2} x^{2}}{k_{\|}^{2}+\eta_{F}^{2} k_{z}^{2}}\right) .
$$

Generally, the damping of bosonic modes with momentum $\mathbf{k}$ is caused by particle-hole excitations close to the part of the Fermi surface that is tangential to $\mathbf{k}$. This is reflected in the momentum dependence of $\langle\nu\rangle$. For the modes with vanishing in-plane momentum, $k_{\|}=0$, only the part of the Fermi surface with azimuthal angle $\theta=\pi / 2$ is involved in the damping processes, such that $\langle\nu\rangle=\nu(0)$. On the other hand, the damping of modes with vanishing $k_{z}=0$ can occur by exciting particle-hole pairs at any part of the Fermi surface and in this case $\langle\nu\rangle$ is a true average over the angle-dependent density of states.

Thus we obtain for the damping function $\Gamma_{k}$, see Eq. (9), for an anisotropic Fermi surface

$$
\Gamma_{k}=\frac{2 v_{F}}{\pi\langle\nu\rangle} \sqrt{k_{\|}^{2}+\left(\eta_{F} k_{z}\right)^{2}}
$$

In the $2 \mathrm{~d}$ regime, damping is dominated by the in-plane momentum, $\Gamma_{k} \sim\left|k_{\|}\right|$, and we obtain the expression advertised in Eq. (12).

\section{Open Fermi surface}

In the limit of a quasi two-dimensional Fermi liquid, the Fermi surface opens as depicted in Fig. 8b. If we neglect the warping of the Fermi-surface cylinder along the momentum $q_{z}$ direction, we end up with a Lindhard function of an effectively $2 \mathrm{~d}$ Fermi system that is, in particular, independent of the longitudinal momentum $k_{z}$. Its dynamic part then has the form

$$
\Pi_{\mathrm{dyn}}\left(k_{\|}, i \omega_{n}\right)=-\nu_{\|} \frac{\left|\omega_{n}\right|}{v_{F} k_{\|}} \sum_{q_{z}}
$$

with the $2 \mathrm{~d}$ density of states $\nu_{\|}$. The integration over the longitudinal momentum $q_{z}$ just yields a multiplicative factor.

If we take the warping into account, we obtain instead the same limiting behavior as that of expression (A4). In the limit $\eta_{F} k_{z} \ll k_{\|}$, where $\eta_{F}$ is again a small parameter representing the strong anisoptropy of the open Fermi surface, the result is essentially unchanged from Eq. (A7) except that $\nu_{\|}$is replaced by an averaged density of states. In the other limit of small in-plane momentum $\eta_{F} k_{z} \gg k_{\|}$, the warping in Fig. $8 \mathrm{~b}$ leads to a $k_{z}$ dependence of the dynamic part of the polarization resulting from particle-hole excitations now concentrated at the center, $q_{z} \approx 0$, and at the edges, $q_{z} \approx \pm \pi / a$, of the Brillouin zone, where the Fermi surface is parallel to $k_{z}$.
${ }^{1}$ H. v. Löhneysen, A. Rosch, M. Vojta, and P. Wölfle, Rev. Mod. Phys. 79, 1015 (2007).

2 J. A. Hertz, Phys. Rev. B 14, 1165 (1976).

3 T. Moriya, Spin Fluctuations in Itinerant Electron Magnetism, Springer-Verlag, Berlin (1985); T. Moriya and T. Takimoto, J. Phys. Soc. Jpn. 64, 960 (1995).

4 A. J. Millis, Phys. Rev. B 48, 7183 (1993).

5 A. Rosch, A. Schröder, O. Stockert, and H. von Löhneysen, Phys. Rev. Lett. 79, 159 (1997).

${ }^{6}$ O. Stockert, H. v. Löhneysen, A. Rosch, N. Pyka, and M. Loewenhaupt, Phys. Rev. Lett. 80, 5627 (1998).

7 O. Trovarelli, C. Geibel, S. Mederle, C. Langhammer, F. M. Grosche, P. Gegenwart, M. Lang, G. Sparn, and F.
Steglich, Phys. Rev. Lett. 85, 626 (2000).

8 P. Gegenwart, J. Custers, Y. Tokiwa, C. Geibel, and F. Steglich, Phys. Rev. Lett. 94, 076402 (2005).

9 Q. Si, S. Rabello, K. Ingersent, and J. L. Smith, Nature (London) 413, 804 (2001); Phys. Rev. B 68, 115103 (2003).

10 P. Coleman, C. Pépin, Q. Si, and R. Ramazashvili, J. Phys: Condens. Matt. 13, 723 (2001).

11 T. Senthil, S. Sachdev, and M. Vojta, Phys. Rev. Lett. 69, 216403 (2003); T. Senthil, M. Vojta, and S. Sachdev, Phys. Rev. B 69, 035111 (2004).

12 L. Zhu, M. Garst, A. Rosch, and Q. Si, Phys. Rev. Lett 91, 066404 (2003).

13 S. E. Sebastian, N. Harrison, C. D. Batista, L. Balicas, 
M. Jaime, P. A. Sharma, N. Kawashima, and I. R. Fisher, Nature 441, 617 (2006).

14 O. Rösch and M. Vojta, Phys. Rev. B 76, 224408 (2007).

15 M. Maltseva and P. Coleman, Phys. Rev. B 72, 174415 (2005).

16 D. Belitz, T. R. Kirkpatrick, and T. Vojta, Phys. Rev. B 55, 9452 (1997).

17 A. Abanov and A. V. Chubukov, Phys. Rev. Lett. 93, 255702 (2004).

18 We assume that the singular terms in the LGW expansion discussed in Refs. 16. 17 are negligible in the $2 \mathrm{~d}$ intermediate-energy regime of the layered system under consideration. (The singularities are not present in the asymptotic 3d AFM regime.) As the singularities are interaction-generated, microscopic details become relevant here. E.g. close to nesting, AF criticality will result already from small interactions, such that the non-LGW regime is expected to be pushed to very small energy scales.

19 P. Jakubczyk, P. Strack, A. A. Katanin, and W. Metzner, Phys. Rev. B 77, 195120 (2008).

20 The standard Hertz model does not describe the ordered phase of a metallic magnet because it does not account for the modified order-parameter dynamics due to gaps in the fermionic spectrum. However, at least for the antiferromagnet in $d>2$, precursor effects of these gaps ("pseudogaps") have been shown to be irrelevant for the critical dynamics, see: A. Rosch, Phys. Rev. B 64, 174407 (2001).

21 A. V. Sologubenko, T. Lorenz, J. A. Mydosh, A. Rosch, K. C. Shortsleeves, and M. M. Turnbull, Phys. Rev. Lett. 100, 137202 (2008).

22 S. Sachdev, Quantum Phase Transitions, Cambridge University Press, Cambridge (1999).

23 A. I. Larkin and D. E. Khmel'nitskii, Zh. Eksperim. i Teor. Fiz. 56, 2087 (1969) [Sov. Phys. JETP 29, 1123 (1969)].

24 F. J. Wegner and E. K. Riedel, Phys. Rev B 7, 248 (1973).

${ }^{25}$ F. Anfuso, M. Garst, A. Rosch, O. Heyer, T. Lorenz, C. Rüegg, and K. Krämer, Phys. Rev. B 77, 235113 (2008).

${ }^{26}$ I. Fischer and A. Rosch, Phys. Rev. B 71, 184429 (2005).
27 The analysis in Ref. 12 missed the sub-leading $T^{3 / 4}$ temperature correction to the thermal expansion for $d=3$, $z=2$.

${ }^{28}$ L. B. Ioffe and A. J. Millis, Phys. Rev. B 51, 16151 (1995).

${ }^{29}$ H. v. Löhneysen, T. Pietrus, G. Portisch, H. G. Schlager, A. Schröder, M. Sieck, and T. Trappmann, Phys. Rev. Lett. 72, 3262 (1994).

30 T. Vojta, Phys. Rev. Lett. 90, 107202 (2003).

31 J. Custers, P. Gegenwart, H. Wilhelm, K. Neumaier, Y. Tokiwa, O. Trovarelli, C. Geibel, F. Steglich, C. Pépin, and P. Coleman, Nature 424, 524 (2003).

32 S. Paschen, T. Lühmann, S. Wirth, P. Gegenwart, O. Trovarelli, C. Geibel, F. Steglich, P. Coleman, and Q. Si, Nature 432, 881 (2004).

33 J. Sichelschmidt, V. A. Ivanshin, J. Ferstl, C. Geibel, and F. Steglich, Phys. Rev. Lett. 91, 156401 (2003).

34 C. Krellner, T. Förster, H. Jeevan, C. Geibel, and J. Sichelschmidt, Phys. Rev. Lett. 100, 066401 (2008).

35 R. Küchler, N. Oeschler, P. Gegenwart, T. Cichorek, K. Neumaier, O. Tegus, C. Geibel, J. A. Mydosh, F. Steglich, L. Zhu, and Q. Si, Phys. Rev. Lett. 91, 066405 (2003).

36 P. Gegenwart, Y. Tokiwa, K. Neumaier, C. Geibel, and F. Steglich, Physica B 359-361, 23 (2005).

37 P. Gegenwart, T. Westerkamp, C. Krellner, Y. Tokiwa, S. Paschen, C. Geibel, F. Steglich, E. Abrahams, and Q. Si, Science 315, 969 (2007).

38 P. Gegenwart, private communication.

39 A. Bianchi, R. Movshovich, I. Vekhter, P. G. Pagliuso, and J. L. Sarrao, Phys. Rev. Lett. 91, 257001 (2003).

40 F. Ronning, C. Capan, E. D. Bauer, J. D. Thompson, J. L. Sarrao, and R. Movshovich, Phys. Rev. B 73, 064519 (2006).

41 J. G. Donath, P. Gegenwart, F. Steglich, E. D. Bauer, and J. L. Sarrao, Phys. Rev. Lett. 100, 136401 (2008).

42 J. Paglione, M. A. Tanatar, D. G. Hawthorn, F. Ronning, R. W. Hill, M. Sutherland, L. Taillefer, and C. Petrovic, Phys. Rev. Lett. 97, 106606 (2006). 